$\omega^{24}$

2. To: (Receiving Organization)

Distribution

5. Proj./Prog./Dept./Div.:

SNF/CSB

8. Originator Remarks:

For approval and release.

11. Receiver Remarks: 11A. Design Baseline Document? [ ] Yes [x] No
3. From: (Originating Organization)

CSB Subproject

6. Design Authority/ Design Agent/Cog. Engr.:

S. A. Krieg

\begin{tabular}{|c|c|c|c|c|c|c|c|c|}
\hline & \multicolumn{4}{|c|}{$\mathrm{N} / \mathrm{A}$} \\
\hline & & & & & \multirow{2}{*}{\multicolumn{4}{|c|}{$\begin{array}{l}\text { 13. Permit/Permit Application No.: } \\
\text { N/A }\end{array}$}} \\
\hline & & & & & & & & \\
\hline & & & & & \multirow{2}{*}{\multicolumn{4}{|c|}{$\begin{array}{l}\text { 14. Required Response Date: } \\
\text { N/A }\end{array}$}} \\
\hline & & & & & & & & \\
\hline 15. & \multicolumn{4}{|c|}{ DATA TRANSMITTED } & (F) & $(\mathrm{G})$ & (H) & (I) \\
\hline $\begin{array}{l}(A) \\
\text { Item } \\
\text { No. }\end{array}$ & (B) Document Drawing No. & $\begin{array}{ll}\text { (C) } \\
\text { Sheet } \\
\text { No. }\end{array}$ & $\begin{array}{l}\text { (D) } \\
\text { Rev. } \\
\text { No. }\end{array}$ & $\begin{array}{c}\text { (E) Title or Description of Data Transmitted } \\
\text { Canister Stor ace Buildin }\end{array}$ & $\begin{array}{l}\text { Approval } \\
\text { Designator }\end{array}$ & $\begin{array}{c}\text { Reason for } \\
\text { Trans- } \\
\text { mitral }\end{array}$ & $\begin{array}{c}\text { Origignator } \\
\text { Dispo- } \\
\text { sition }\end{array}$ & $\begin{array}{l}\text { Receciver } \\
\text { Dispo- } \\
\text { sition }\end{array}$ \\
\hline 1 & SNF-6253 & N/A & 0 & $\begin{array}{l}\text { Acceptance for Beneficial Use } \\
\text { Index - Roadmap of Supporting } \\
\text { Documents }\end{array}$ & $\mathrm{E}, \mathrm{S}, \mathrm{Q}$ & 1,2 & 1 & \\
\hline & & & & & & & & \\
\hline & & & & & & & & \\
\hline & & & & & & & & \\
\hline & & & & & & & & \\
\hline & & & & & & & & \\
\hline & & & & & & & & \\
\hline & & & & & & & & \\
\hline & & & & & & & & \\
\hline
\end{tabular}

\begin{tabular}{|c|c|c|c|c|}
\hline \multicolumn{3}{|l|}{16.} & \multicolumn{2}{|c|}{ KEY } \\
\hline Approval Designator (F) & & Reason for Transmittal (G) & & tion $(\mathrm{H}) \&(\mathrm{I})$ \\
\hline $\begin{array}{l}\text { E, S, Q, D or N/A } \\
\text { (see WHC-CM-3-S, Sec.12.7) }\end{array}$ & $\begin{array}{l}\text { 1. Approval } \\
\text { 2. Release } \\
\text { 3. Information }\end{array}$ & $\begin{array}{l}\text { 4. Review } \\
\text { 5. Post-Review } \\
\text { 6. Dist. (Receipt Acknow. Required) }\end{array}$ & $\begin{array}{l}\text { 1. Approved } \\
\text { 2. Approved w/comment } \\
\text { 3. Disapproved w/comment }\end{array}$ & $\begin{array}{l}\text { 4. Reviewed no/comment } \\
\text { 5. Reviewed w/comment } \\
\text { 6. Receipt acknowledged }\end{array}$ \\
\hline
\end{tabular}

(See Approval Designator for required signatures)

\begin{tabular}{|c|c|c|c|c|c|}
\hline $\begin{array}{l}\text { (G) } \\
\text { Reason }\end{array}$ & $\begin{array}{l}\text { (H) } \\
\text { Disp. }\end{array}$ & $\begin{array}{lll}\text { (J) Name } & \text { (K) Signature (L) Date (M) MSIN }\end{array}$ & $\begin{array}{c}\text { (G) } \\
\text { Reason }\end{array}$ & $\begin{array}{l}\text { (H) } \\
\text { Disp. }\end{array}$ & (K) Signature (L) Date (M) MSIN \\
\hline 1 & t & CSB DA S. A. Krieg Xfl. Krieg, 5-1-00 & 1 & & Sofety R. L. Garrett $2 / 2$ \\
\hline 1 & 1 & CTS DA D. M. Chenault DChedf $5 y-00$ & 1 & & Env. J. E. Turnbaugh S S-Twule \\
\hline$\overline{1}$ & 1 & MCO DA L. H. Goldmann Guin 1/F/gemom-4/26/2000 & 1 & & Facility Ops Mgr. O. M.Serrano/ \\
\hline 1 & $\bar{z}$ & MHM DA C. E. Swenson haug thenem $4 / 27$ bs & 4 & & Ops Eng. J. O. Bilskis \\
\hline 1 & 1 & Cog. Eng. D. M. Black $\mathrm{D} / \mathrm{M} / \mathrm{R} / \mathrm{k}$, 4.26 .00 & 1 & 1 & Ops Eng. M \\
\hline 1 & & 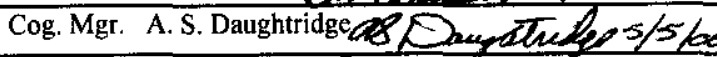 & 1 & 1 & CTS \& MCO Proj. Mgr. \\
\hline 1 & 1 & QA S. S. Moss Btemher Batt Moote 4.27-2000 & 1 & 1 & CSB Start \\
\hline
\end{tabular}
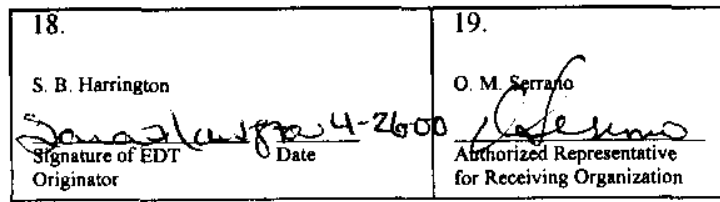

$\frac{\infty 00508}{\text { Date }}$

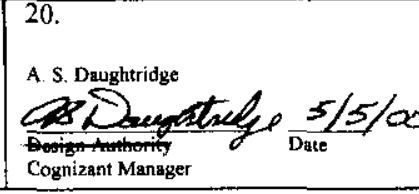

Cognizant Manager
21. DOE APPROVAL (if required)

Ctrl. No.

[] Approved

[] Approved w/comments N/A

[] Disapproved w/comments 
SNF-6253

Revision 0

\section{Canister Storage Building Acceptance for Beneficial Use Index - Roadmap of Supporting Documents}

Prepared for the U.S. Department of Energy

Assistant Secretary for Environmental Management

Project Hanford Management Contractor for the

U.S. Department of Energy under Contract DE-AC06-96RL13200

Fluor Hanford

P.o. Box 1000

Richland, Washington 
SNF-6253

Revision 0

EDT 628696

\section{Canister Storage Building Acceptance for Beneficial Use Index - Roadmap of Supporting Documents}

D. M. Black

Fluor Federal Services

J. O. Bilskis

Fluor Hanford

$$
\text { pages }-55
$$

Date Published

May 2000

Prepared for the U.S. Department of Energy

Assistant Secretary for Environmental Management

Project Hanford Management Contractor for the

U.S. Department of Energy under Contract DE-AC06-96RL13200

\section{Fluor Hanford}

P.O. Box 1000

Richland, Washington
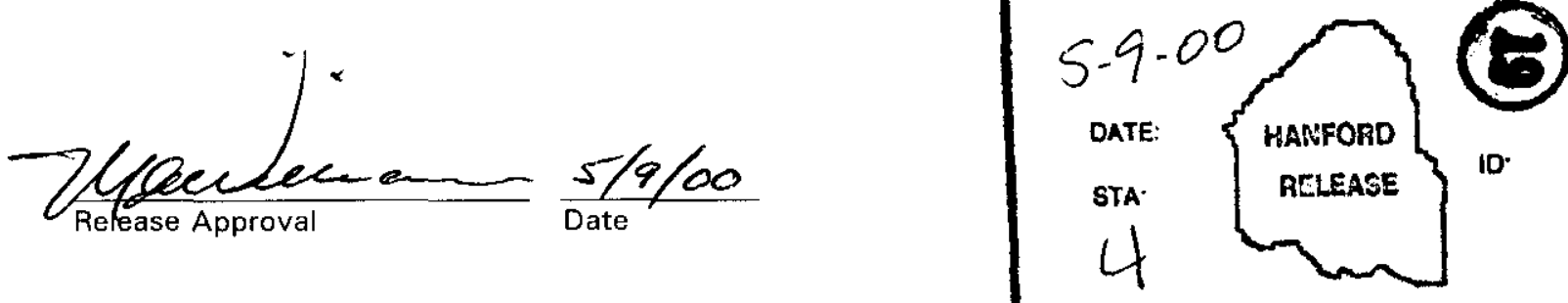
TRADEMARK DISCLAIMER

Reference herein to any specific commercial product, process,

or service by trade name, trademark, manufacturer, or

otherwise, does not necessarily constitute or imply its

endorsement, recommendation, or favoring by the United

States Government or any agency thereof or its contractors or subcontractors.

This report has been reproduced from the best available copy. 
SNF-6253 REV 0

CANISTER STORAGE BUIDLING

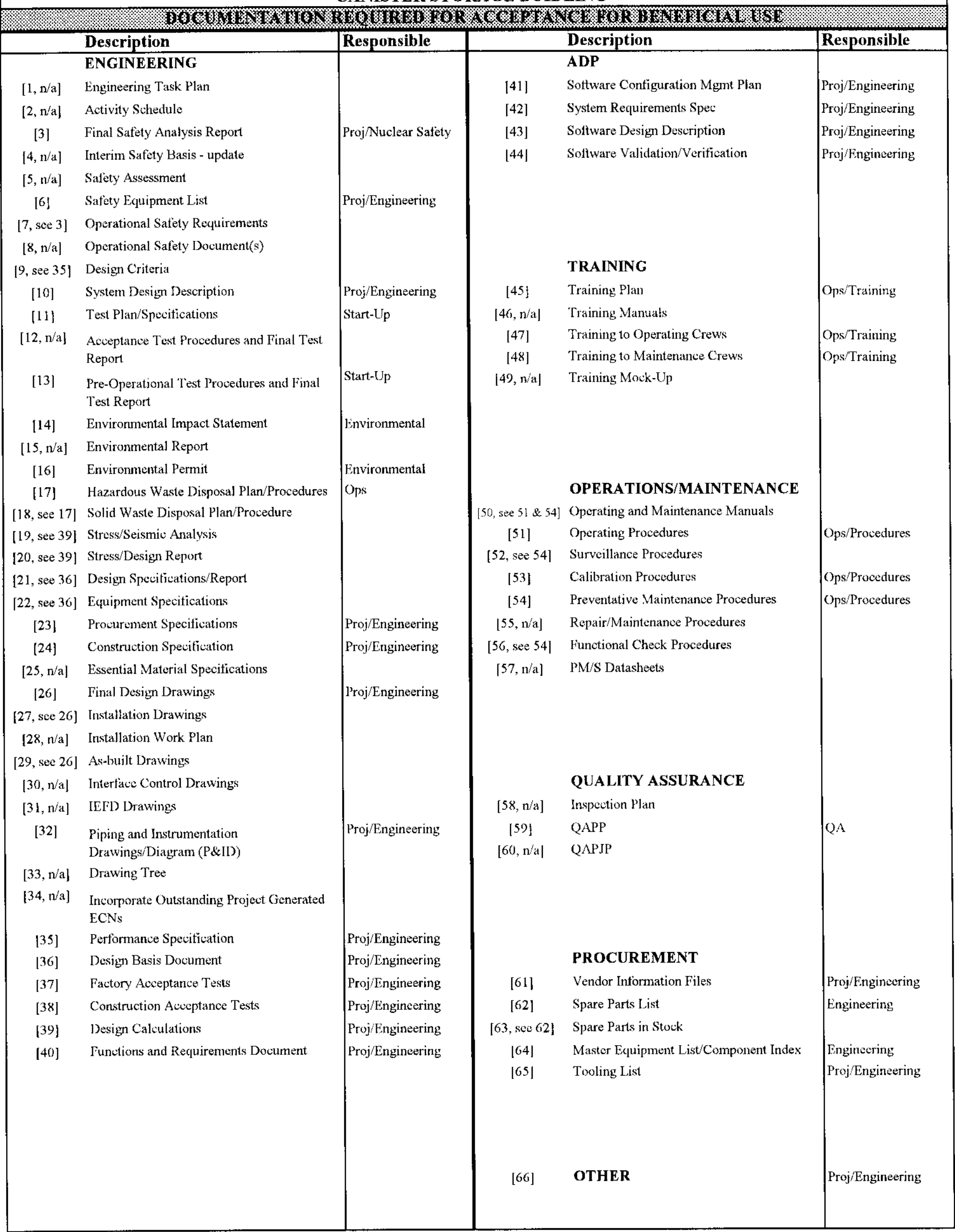


SNF-6253 REV 0

MULTI-CANISTER OVERPACKS AND BASKETS

\begin{tabular}{|c|c|c|c|c|c|}
\hline \multicolumn{6}{|c|}{ MULTI-CANISTER OVERPACKS AND BASKETS } \\
\hline \multicolumn{6}{|c|}{ 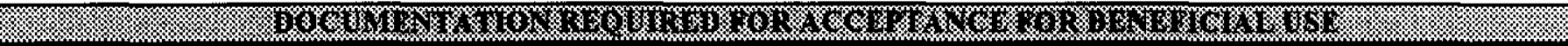 } \\
\hline & Description & Responsible & & Description & Responsible \\
\hline & ENGINEERING & & & ADP & \\
\hline$[1, \mathbf{n} / \mathbf{a}]$ & Engineering Task Plan & & {$[41, n / a]$} & Software Configuration Mgmt Plan & \\
\hline$[2, n / a]$ & Activity Schedule & & {$[42, n / a]$} & System Requirements Spec & \\
\hline [3] & Final Safety Analysis Report & Proj/Nuclear Safety & {$[43, n / a]$} & Software Design Description & \\
\hline$[4, \mathbf{n} / \mathbf{a}]$ & Interim Safety Basis - update & & [44] & Software Validation/Verification & Proj/Engineering \\
\hline$[5, n / a]$ & Safety Assessment & & & & \\
\hline$[6$, see 3$]$ & Safety Equipment List & & & & \\
\hline$\{7$, see 3$\}$ & Operational Safety Requirements & & & & \\
\hline$[8, \mathrm{n} / \mathrm{a}]$ & Operational Safety Document(s) & & & & \\
\hline [9] & Design Criteria & Proj/Engineering & & TRAINING & \\
\hline$[10, \mathrm{n} / \mathrm{a}]$ & System Design Description & & {$[45, \mathrm{n} / \mathrm{a}]$} & Training Plan & \\
\hline [11] & Test Plan/Specifications & Proj/Engineering & {$[46, \mathrm{n} / \mathrm{a}]$} & Training Manuals & \\
\hline$[12, \operatorname{see} 61]$ & Acceptance Test Procedures and Final Test & & {$[47, \mathrm{n} / \mathrm{a}]$} & Training to Operating Crews & \\
\hline & Report & & {$[48, \mathrm{n} / \mathrm{a}]$} & Training to Maintenance Crews & \\
\hline$[13, \mathbf{n} / \mathbf{a}]$ & $\begin{array}{l}\text { Pre-Operational Test Procedures and Final } \\
\text { Test Report }\end{array}$ & & {$[49, \mathrm{n} / \mathrm{a}]$} & Training Mock-Up & \\
\hline$[14, \mathbf{n} / \mathbf{a}]$ & Environmental Impact Statement & & & & \\
\hline$[15, \mathrm{n} / \mathrm{a}]$ & Environmental Report & & & & \\
\hline$[16, \mathrm{n} / \mathrm{a}]$ & Environmental Permit & & & & \\
\hline$[17, \mathrm{n} / \mathrm{a}]$ & Hazardous Waste Disposal Plan/Procedures & & & OPERATIONS/MAINTENANCE & \\
\hline$[18, \mathrm{n} / \mathrm{a}]$ & Solid Waste Disposal Plan/Procedure & & [50] & Operating and Maintenance Manuals & Proj/Engineering \\
\hline$[19$, see 20$]$ & Stress/Seismic Analysis & & {$[51, \mathrm{n} / \mathrm{a}]$} & Operating Procedures & \\
\hline [20] & Stress/Design Report & Proj/Engineering & {$[52, \mathrm{n} / \mathrm{a}]$} & Surveillance Procedures & \\
\hline$[21$, see 9] & Design Specifications/Report & & {$[53, \mathrm{n} / \mathrm{a}]$} & Calibration Procedures & \\
\hline$[22, \mathbf{n} / \mathbf{a}]$ & Equipment Specifications & & {$[54, \mathrm{n} / \mathrm{a}]$} & Preventative Maintenance Procedures & \\
\hline [23] & Procurement Specifications & Proj/Engineering & {$[55, \mathrm{n} / \mathrm{a}]$} & Repair/Maintenance Procedures & \\
\hline$[24, n / a]$ & Construction Specification & & {$[56, \mathrm{n} / \mathrm{a}]$} & Functional Check Procedures & \\
\hline$[25$, see 23$]$ & Essential Material Specifications & & {$[57, \mathrm{n} / \mathrm{a}]$} & PM/S Datasheets & \\
\hline [26] & Final Design Drawings & Proj/Engineering & & & \\
\hline$[27, n / a]$ & Installation Drawings & & & & \\
\hline$[28, \mathrm{n} / \mathrm{a}]$ & Installation Work Plan & & & & \\
\hline$[29$, see 61$]$ & As-built Drawings & & & & \\
\hline$[30, \mathbf{n} / \mathbf{a}]$ & Interface Control Drawings & & & QUALITY ASSURANCE & \\
\hline$[31, \mathbf{n} / \mathbf{a}]$ & IEFD Drawings & & {$[58, \operatorname{see} 61]$} & Inspection Plan & \\
\hline$[32, n / a]$ & Piping and Instrumentation & & [59] & QAPP & QA \\
\hline & Drawings/Diagram (P\&ID) & & {$[60, \mathrm{n} / \mathrm{a}]$} & QAPJP & \\
\hline$[33$, see 26$]$ & Drawing Tree & & & & \\
\hline$\{34, n / a]$ & $\begin{array}{l}\text { Incorporate Outstanding Project Generated } \\
\text { ECNs }\end{array}$ & & & & \\
\hline$[35$, see 9] & Performance Specification & & & & \\
\hline$[36$, see 9] & Design Basis Document & & & PROCUREMENT & \\
\hline$[37, \mathrm{n} / \mathrm{a}]$ & Factory Acceptance Tests & & {$[61]$} & Vendor Information Files & Proj/Engineering \\
\hline$[38, n / a]$ & Construction Acceptance Tests & & {$[62$, see 50$]$} & Spare Parts List & \\
\hline$[39$, see 20$]$ & Design Calculations & & {$[63$, see 50$]$} & Spare Parts in Stock & \\
\hline [40] & Functions and Requirements Document & Proj/Engineering & {$[64, n / a]$} & Component Index & \\
\hline & & & {$[65, \mathrm{n} / \mathrm{a}]$} & Tooling List & \\
\hline & & & [66] & OTHER & Project \\
\hline
\end{tabular}


SNF-6253 REV 0

\begin{tabular}{|c|c|c|c|c|c|}
\hline \multicolumn{6}{|c|}{ CASK TRANSPORT SYSTEM } \\
\hline \multicolumn{6}{|c|}{ 1 } \\
\hline & Description & Responsible & & Description & Responsible \\
\hline & ENGINEERING & & & ADP & \\
\hline$[1, n / a\}$ & Engineering Task Plan & & {$[41, \mathrm{n} / \mathbf{a}]$} & Software Configuration Mgmt Plan & \\
\hline$[2, \mathrm{n} / \mathrm{a}]$ & Activity Schedule & & {$[42, n / a]$} & System Requirements Spec & \\
\hline [3] & Final Safety Analysis Report & Proj/Nuclear Safety & {$[43, n / a]$} & Software Design Description & \\
\hline$[4, \mathbf{n} / \mathbf{a}]$ & Interim Safety Basis - update & & {$[44, \mathrm{n} / \mathrm{a}]$} & Software Validation/Verification & \\
\hline$[5, \mathrm{n} / \mathrm{a}]$ & Safety Assessment & & & & \\
\hline$[6$, see 3$]$ & Safety Equipment List & & & & \\
\hline$[7$, see 3] & Operational Safety Requirements & & & & \\
\hline$[8, \mathbf{n} / \mathbf{a}]$ & Operational Safety Document(s) & & & & \\
\hline [9] & Design Criteria & Proj/Engineering & & TRAINING & \\
\hline [10] & System Design Description & Proj/Engineering & {$[45]$} & Training Plan & Ops/Training \\
\hline$[11]$ & Test Plan/Specifications & Proj/Engineering & {$[46, n / a]$} & Training Manuals & \\
\hline$[12$, see 11$]$ & Acceptance Test Procedures and Final Test & & {$[47]$} & Training to Operating Crews & Ops/Training \\
\hline & Report & & {$[48, \mathfrak{n} / \mathbf{a}]$} & Training to Maintenance Crews & \\
\hline$[13$, see 11$]$ & $\begin{array}{l}\text { Pre-Operational Test Procedures and Final } \\
\text { Test Report }\end{array}$ & & {$[49, n / a]$} & Training Mock-Up & \\
\hline$[14, \mathfrak{n} / \mathrm{a}]$ & Environmental Impact Statement & & & & \\
\hline$[15, \mathrm{n} / \mathrm{a}]$ & Environmental Report & & & & \\
\hline$[16, n / a]$ & Environmental Permit & & & & \\
\hline$[17, \mathrm{n} / \mathrm{a}]$ & Hazardous Waste Disposal Plan/Procedures & & & OPERATIONS/MAINTENANCE & \\
\hline$[18, \mathbf{n} / \mathbf{a}]$ & Solid Waste Disposal Plan/Procedure & & {$[50]$} & Operating and Maintenance Manuals & Proj/Engineering \\
\hline$[19$, see 20] & Stress/Seismic Analysis & & {$[51]$} & Operating Procedures & Ops/Procedures \\
\hline$[20]$ & Stress/Design Report & Proj/Engineering & {$[52$, see 54$]$} & Surveillance Procedures & \\
\hline$[21$, see 10$]$ & Design Specifications/Report & & {$[53, n / a]$} & Calibration Procedures & \\
\hline$[22$, see 10$]$ & Equipment Specifications & & {$[54]$} & Preventative Maintenance Procedures & Ops/Procedures \\
\hline$[23, n / a]$ & Procurement Specifications & & {$[55$, see 54$]$} & Repair/Maintenance Procedures & \\
\hline$[24, \mathrm{n} / \mathrm{a}]$ & Construction Specification & & {$[56, \mathrm{n} / \mathrm{a}]$} & Functional Check Procedures & \\
\hline$[25$, see 11$]$ & Essential Material Specifications & & {$[57, \mathrm{n} / \mathrm{a}]$} & PM/S Datasheets & \\
\hline$[26]$ & Final Design Drawings & Proj/Engineering & & & \\
\hline$[27, \sec 9 \& 26]$ & Installation Drawings & & & & \\
\hline$[28, \mathrm{n} / \mathrm{a}]$ & Installation Work Plan & & & & \\
\hline$[29$, see 11$]$ & As-built Drawings & & & & \\
\hline$[30, n / a]$ & Interface Control Drawings & & & QUALITY ASSURANCE & \\
\hline$[31, \mathbf{n} / \mathbf{a}]$ & IEFD Drawings & & {$[58, \operatorname{see} 61]$} & Inspection Plan & \\
\hline [32] & Piping and Instrumentation & Proj/Engineering & {$[59]$} & QAPP & QA \\
\hline & Drawings/Diagram (P\&ID) & & {$[60, n / a]$} & QAPJP & \\
\hline$[33, n / a]$ & Drawing Tree & & & & \\
\hline$[34, n / a]$ & $\begin{array}{l}\text { Incorporate Outstanding Project Generated } \\
\text { ECNs }\end{array}$ & & & & \\
\hline$[35$, see 9$]$ & Performance Specification & & & & \\
\hline$[36$, see 9$]$ & Design Basis Document & & & PROCUREMENT & \\
\hline$[37, \mathrm{n} / \mathrm{a}]$ & Factory Acceptance Tests & & [61] & Vendor Information Files & Proj/Engineering \\
\hline$[38, \mathrm{n} / \mathrm{a}]$ & Construction Acceptance Tests & & {$[62]$} & Spare Parts List & Proj/Engineering \\
\hline$[39$, see 20] & Design Calculations & & {$[63$, see 62$]$} & Spare Parts in Stock & \\
\hline$[40$, see 20$\}$ & Functions and Requirements Document & & $\begin{array}{c}{[64]} \\
{[65, \mathrm{n} / \mathrm{a}]}\end{array}$ & $\begin{array}{l}\text { Component Index } \\
\text { Tooling List }\end{array}$ & Engineering \\
\hline & & & {$[66]$} & OTHER & Proj/Engineering \\
\hline
\end{tabular}




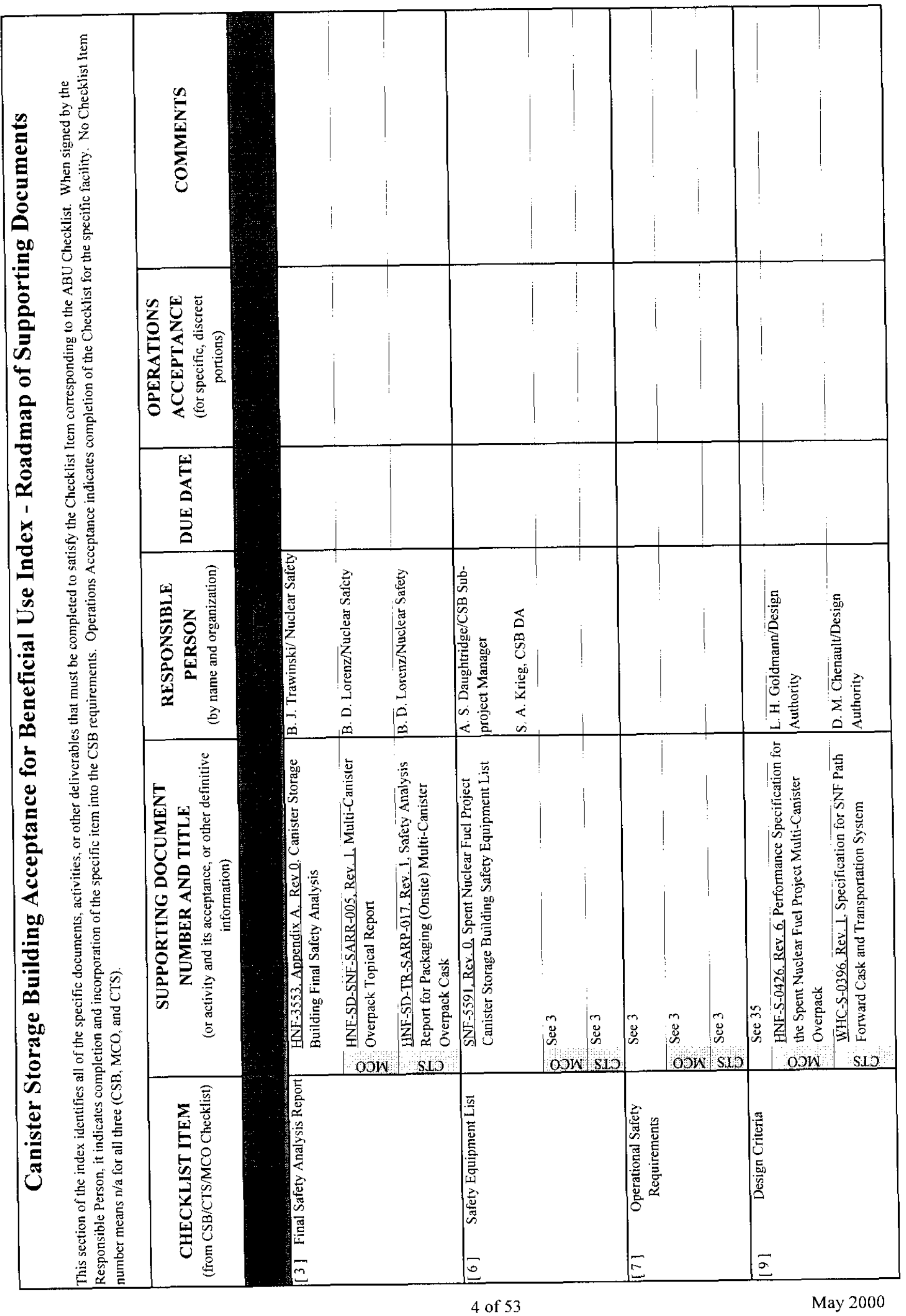


SNF-6253 REV 0

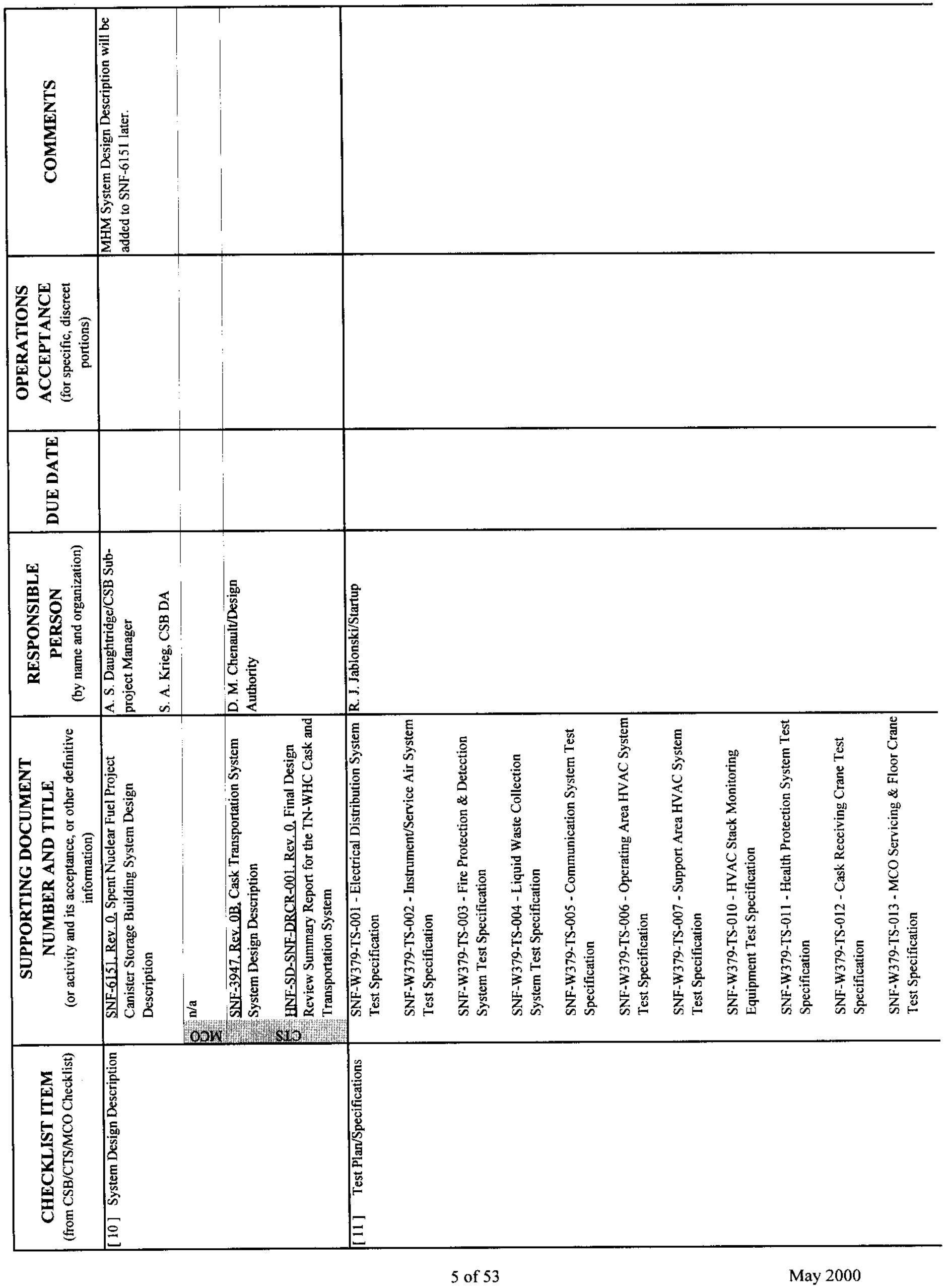




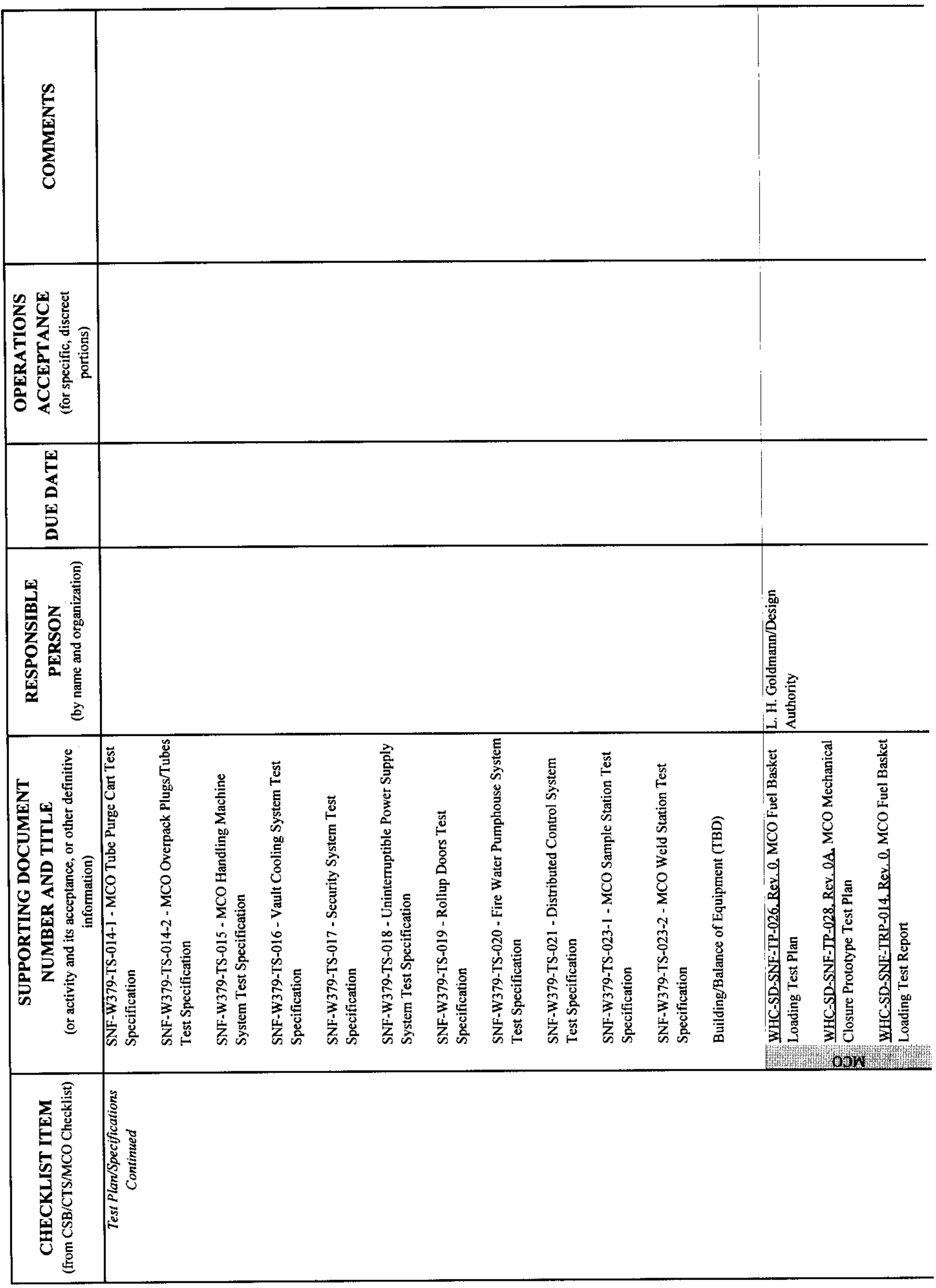


SNF-6253 REV 0

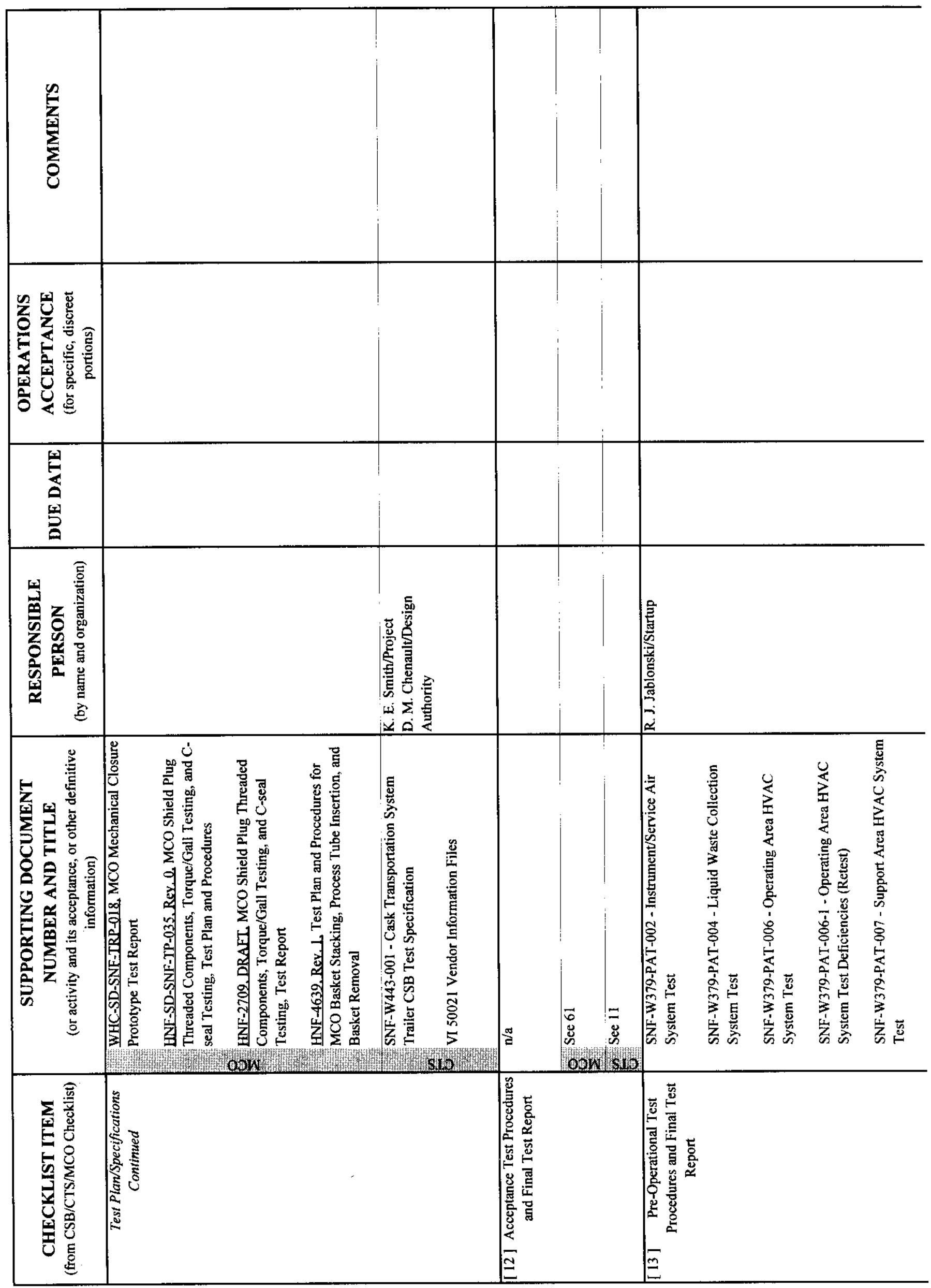




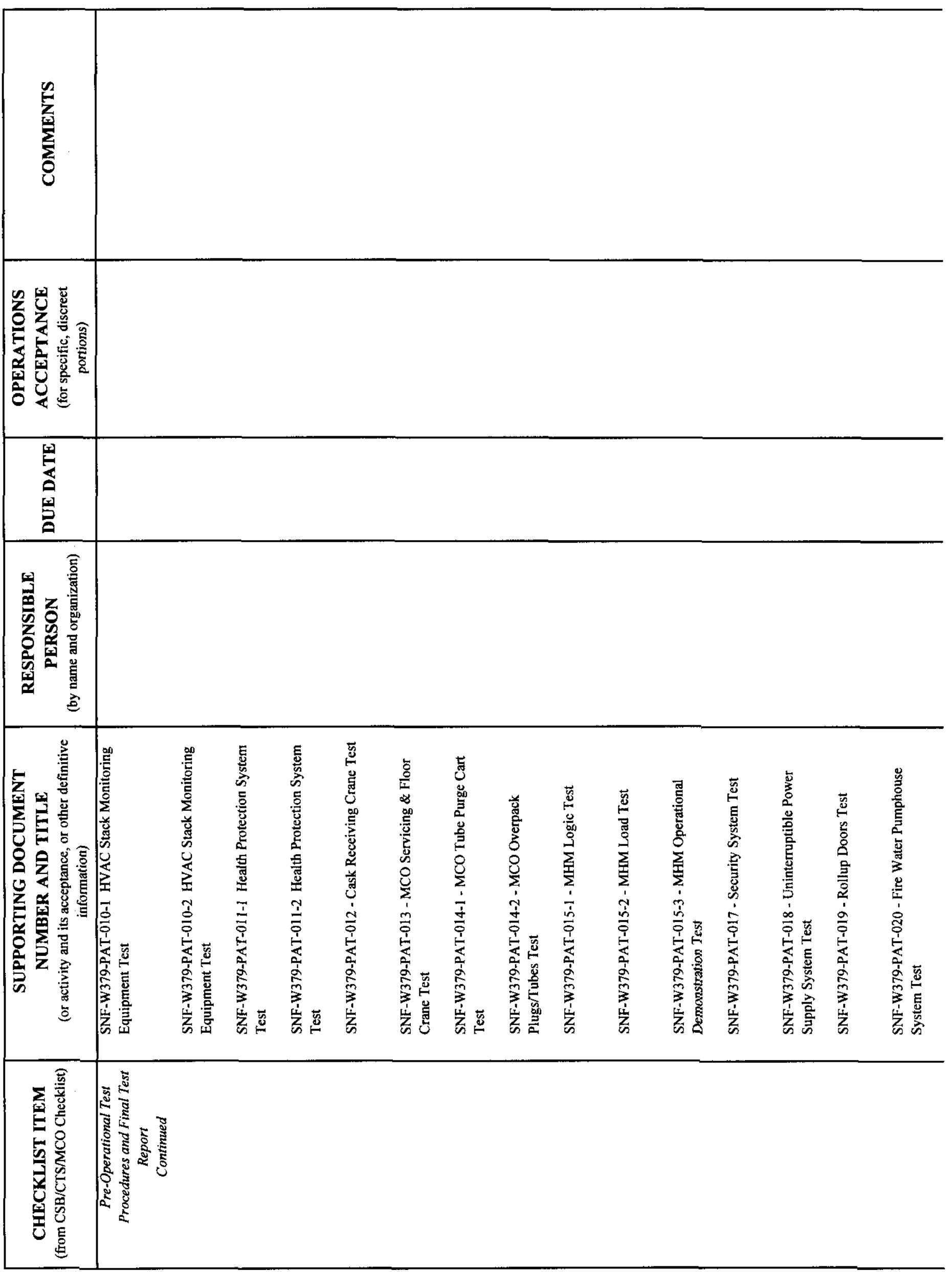




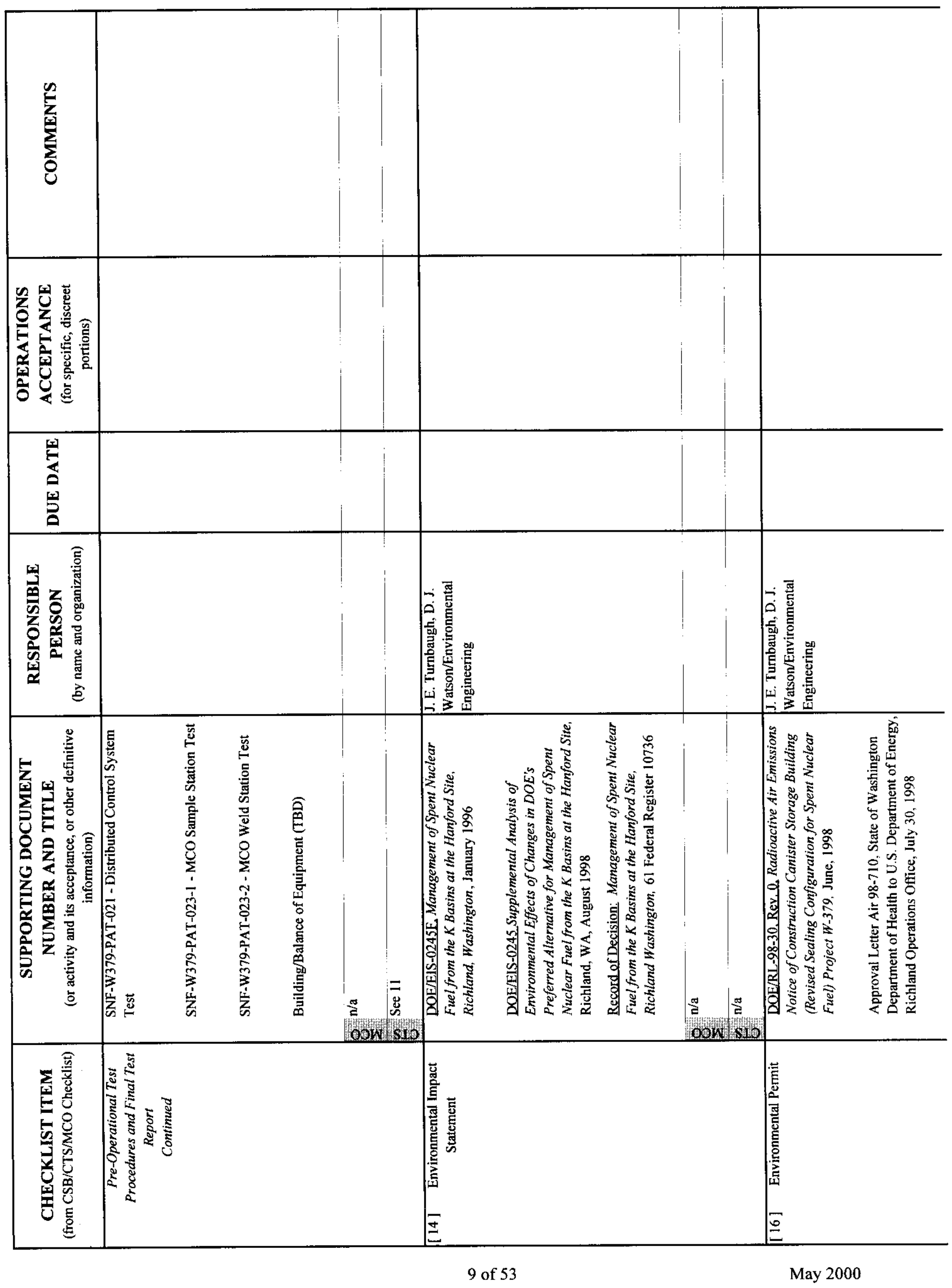


SNF-6253 REV 0

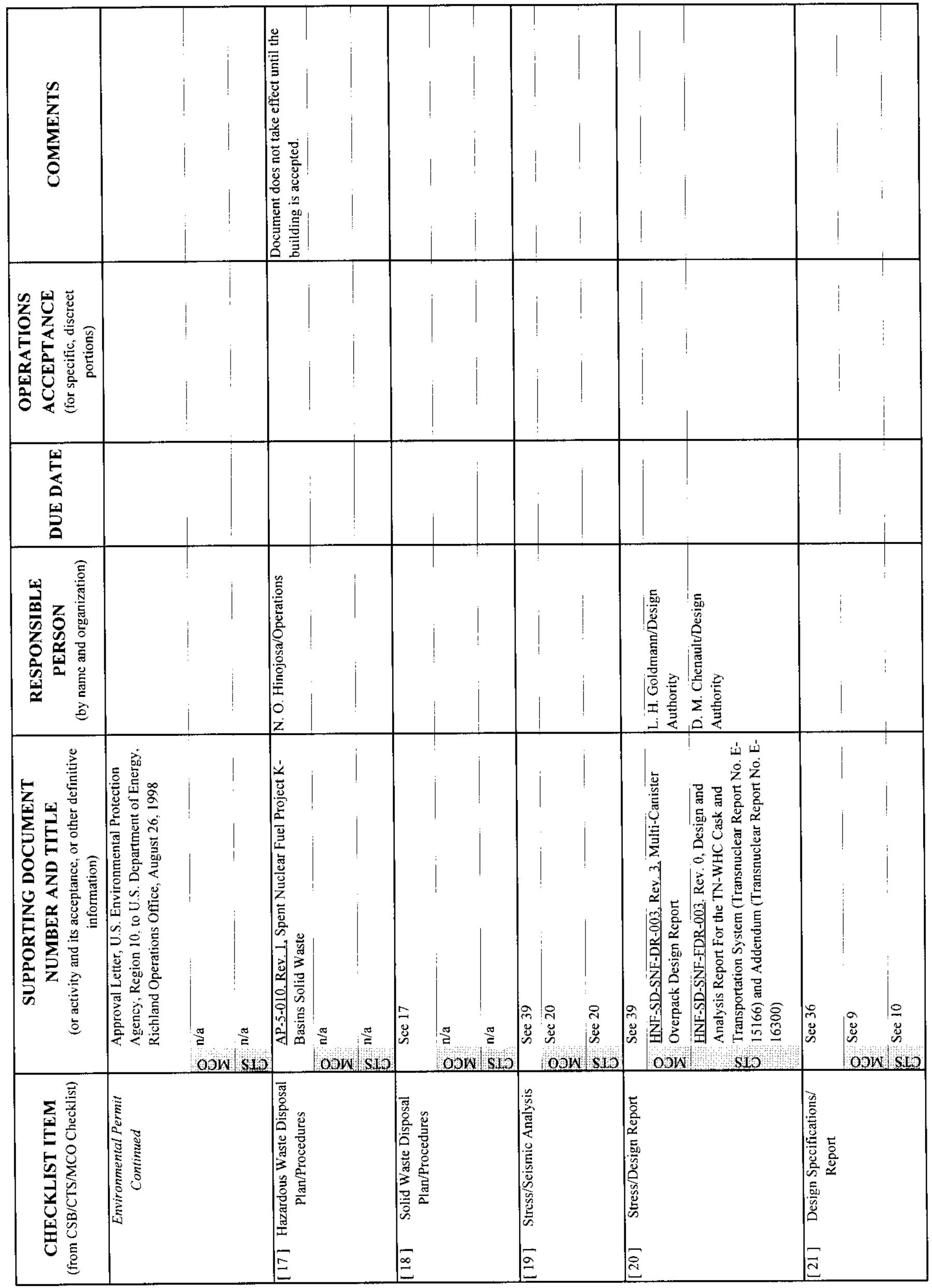


SNF-6253 REV 0

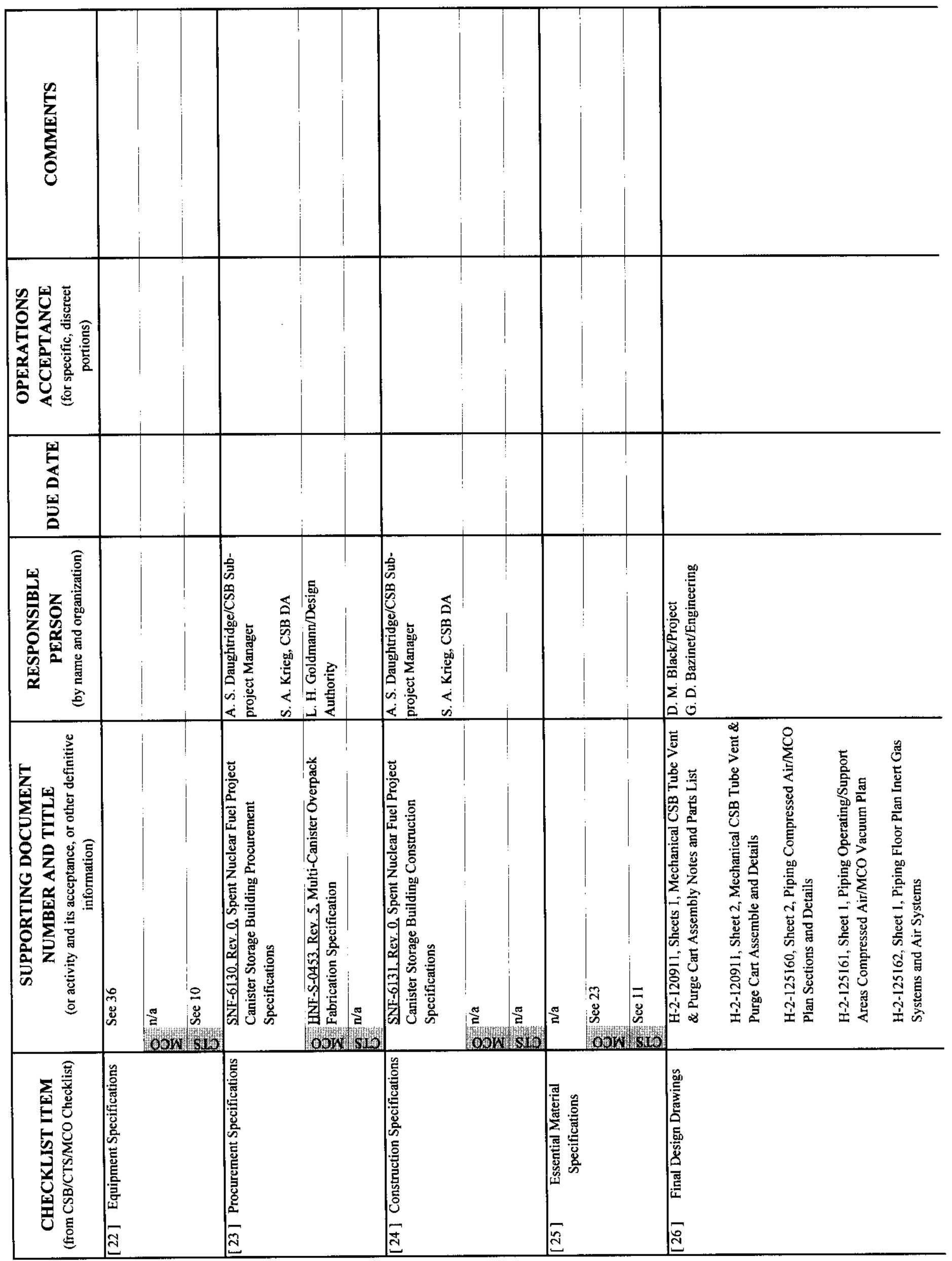




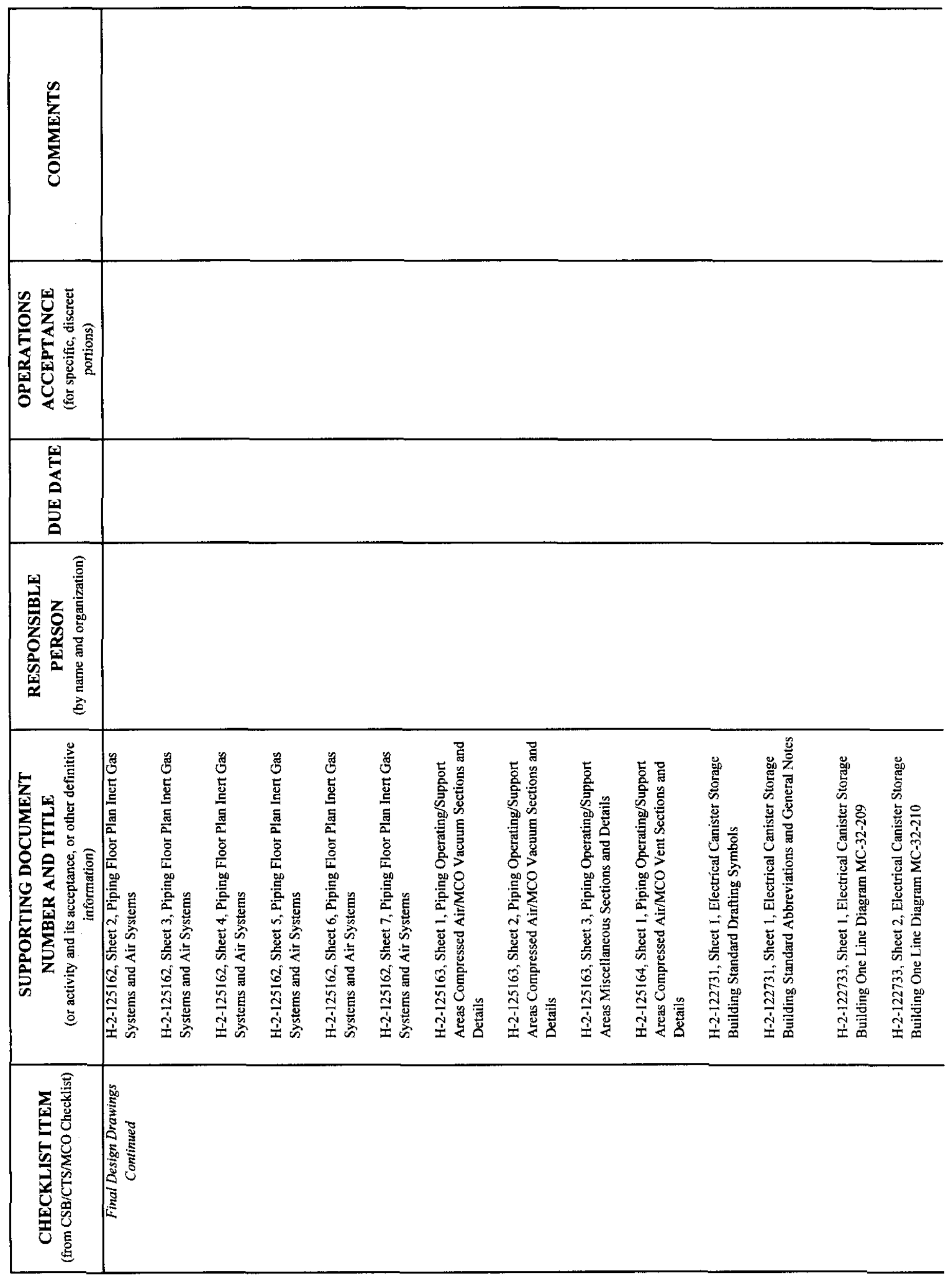




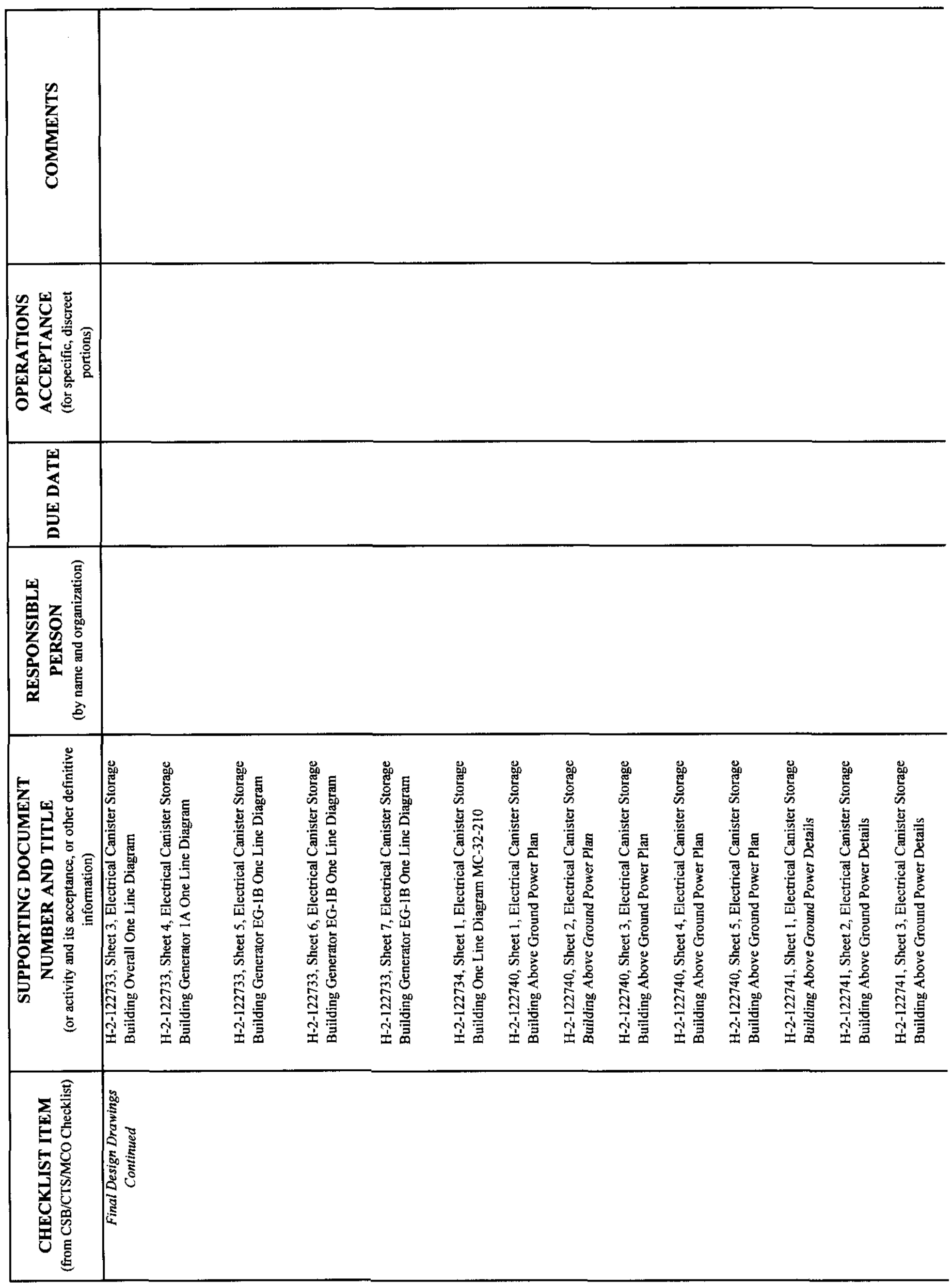




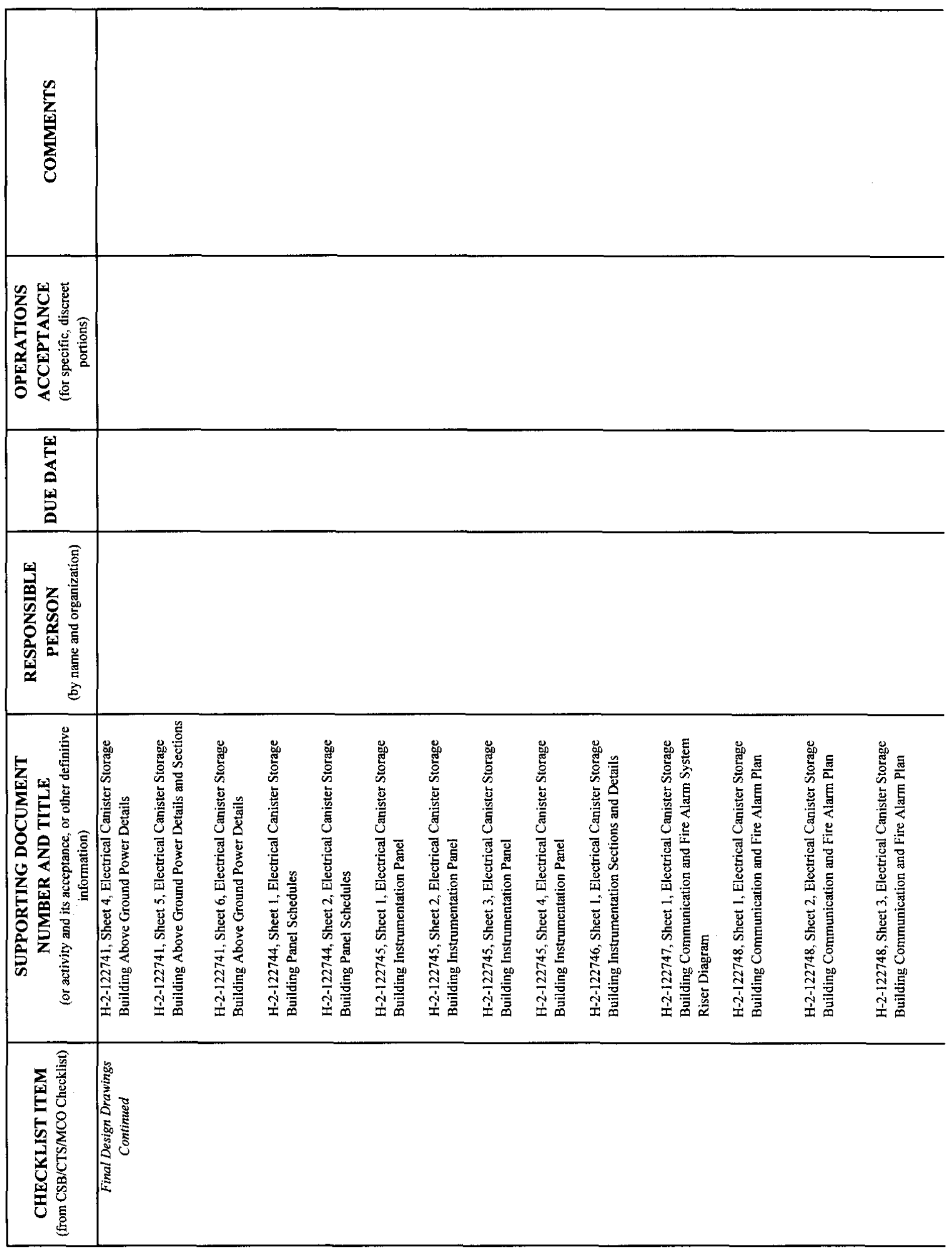


SNF-6253 REV 0

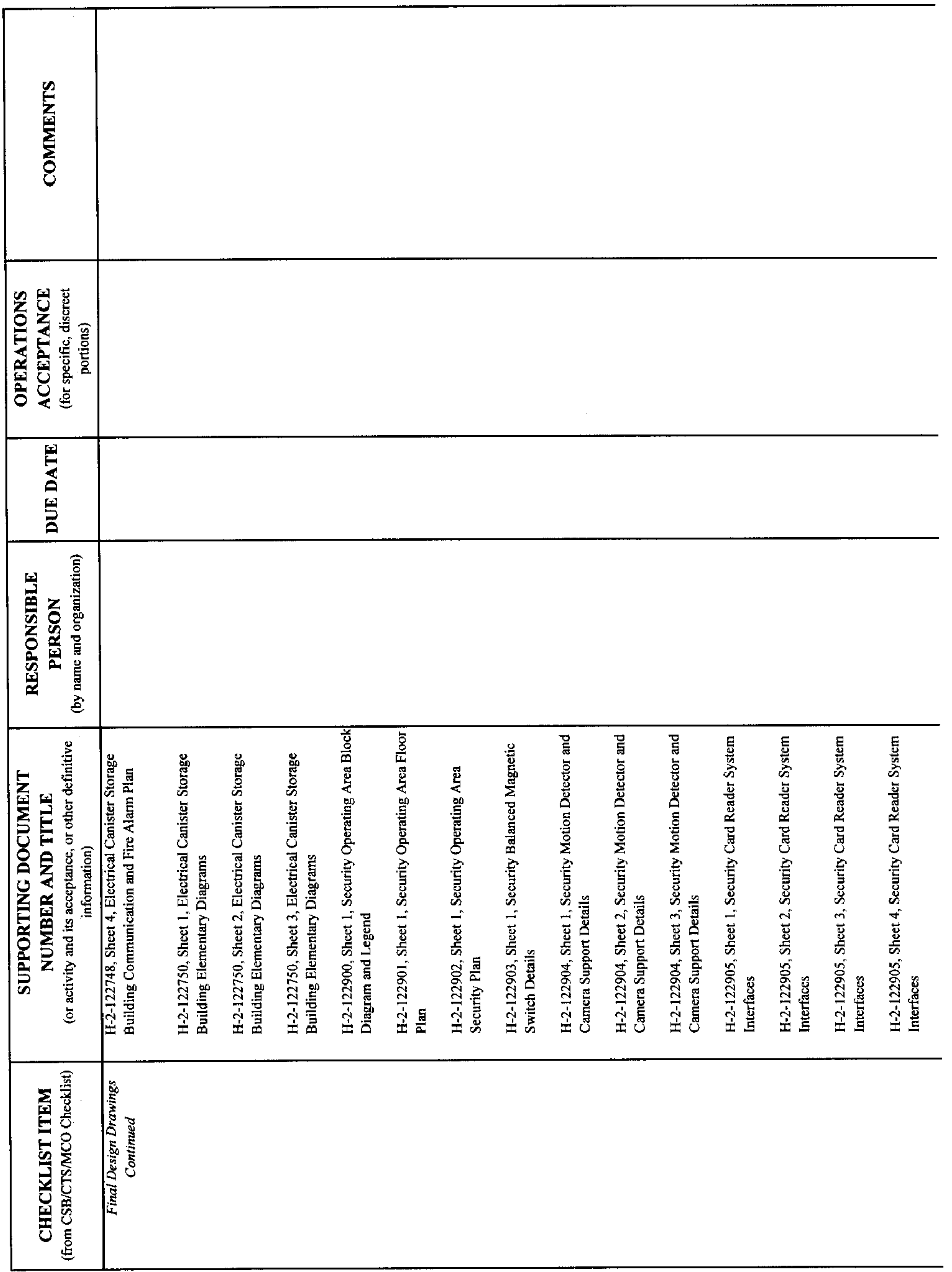


SNF-6253 REV 0

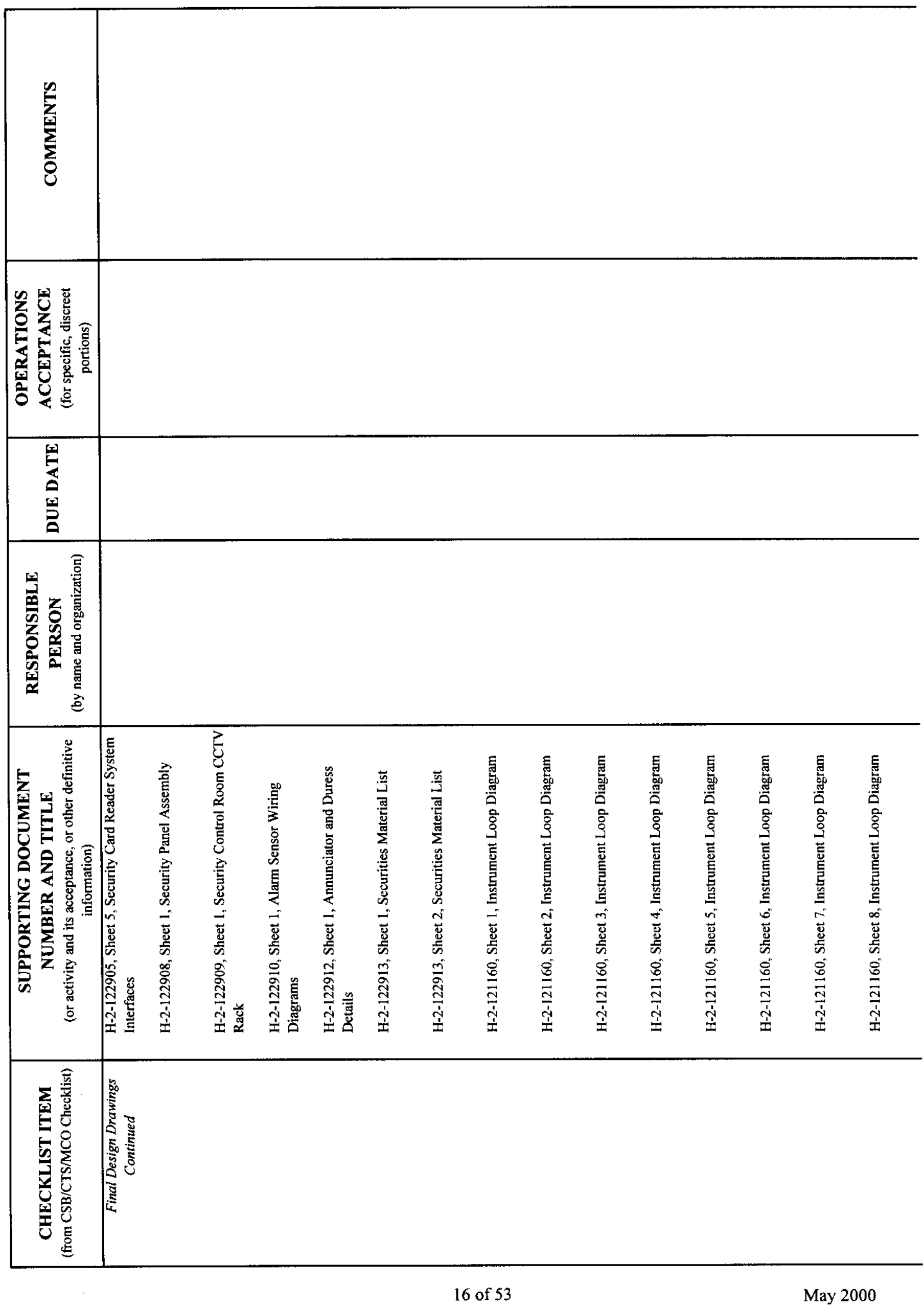


SNF-6253 REV 0

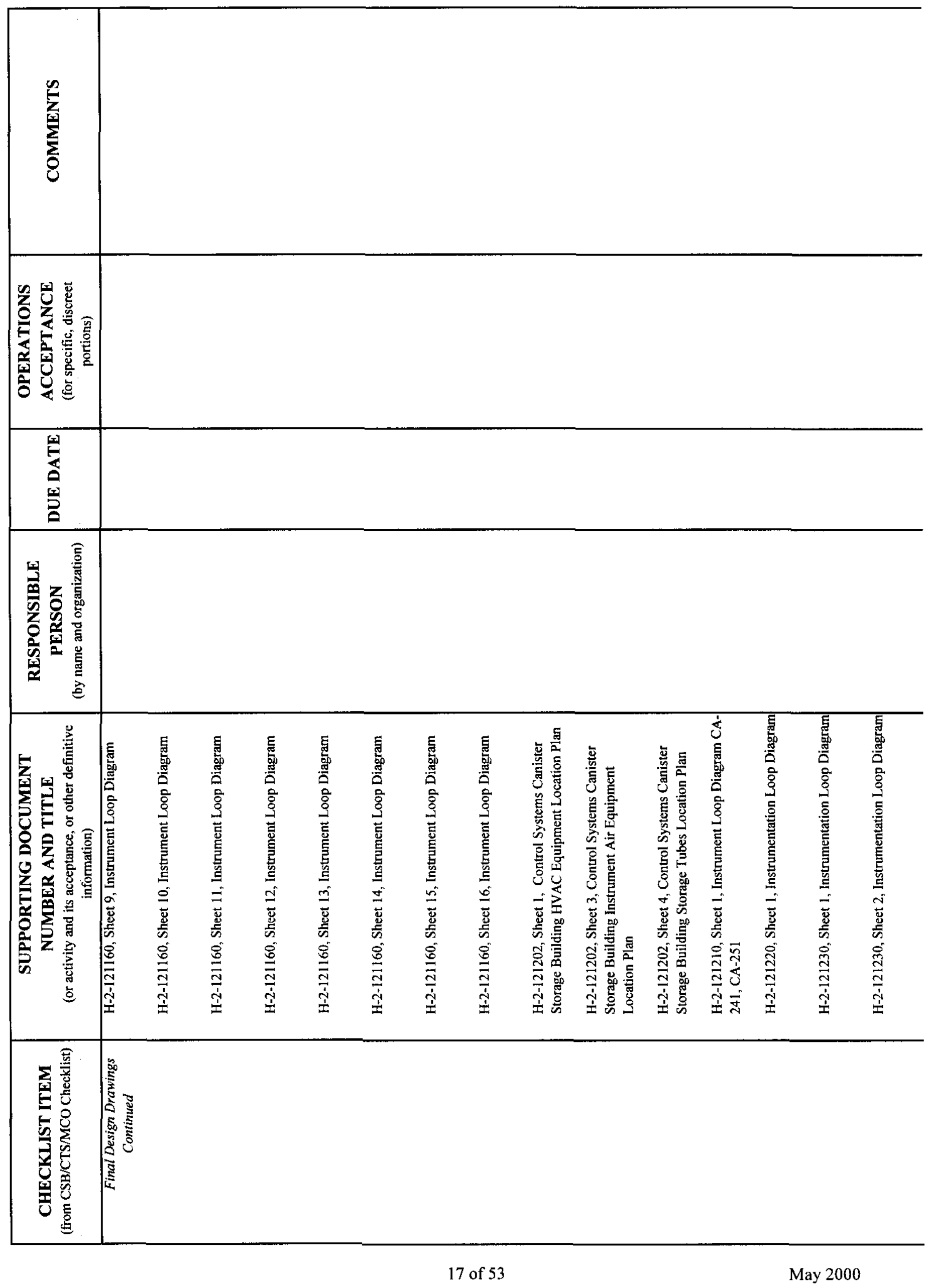




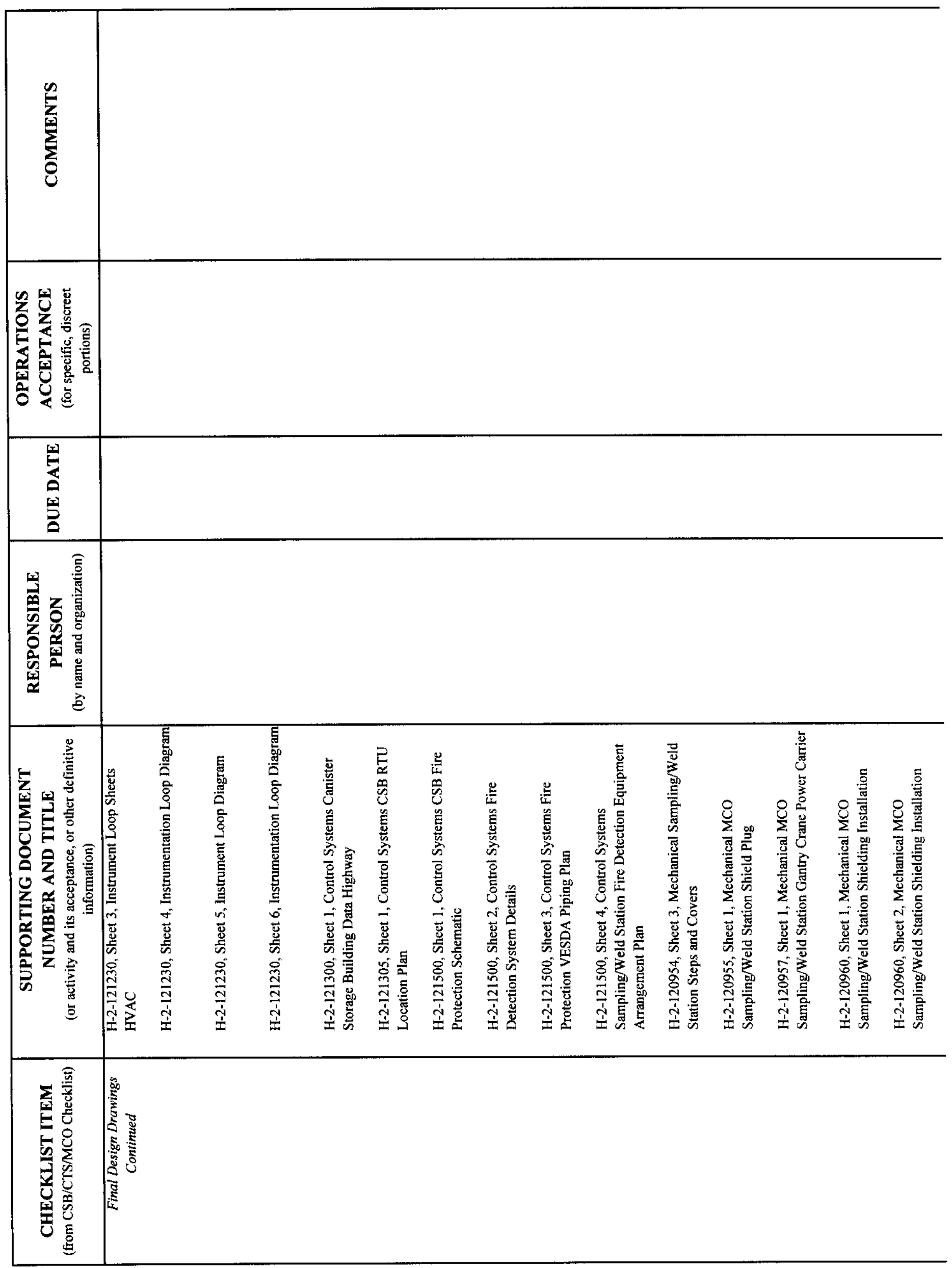


SNF-6253 REV 0

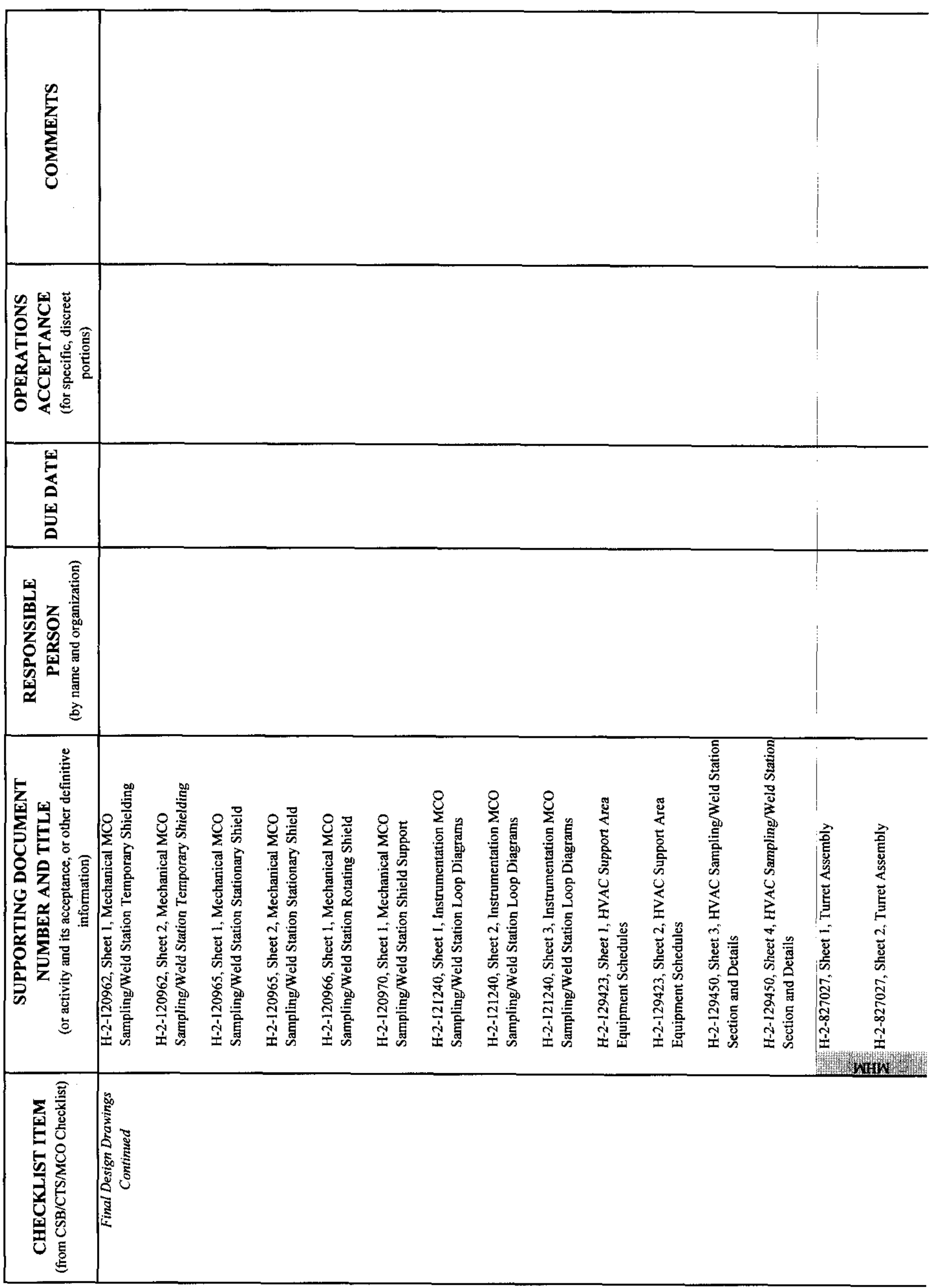




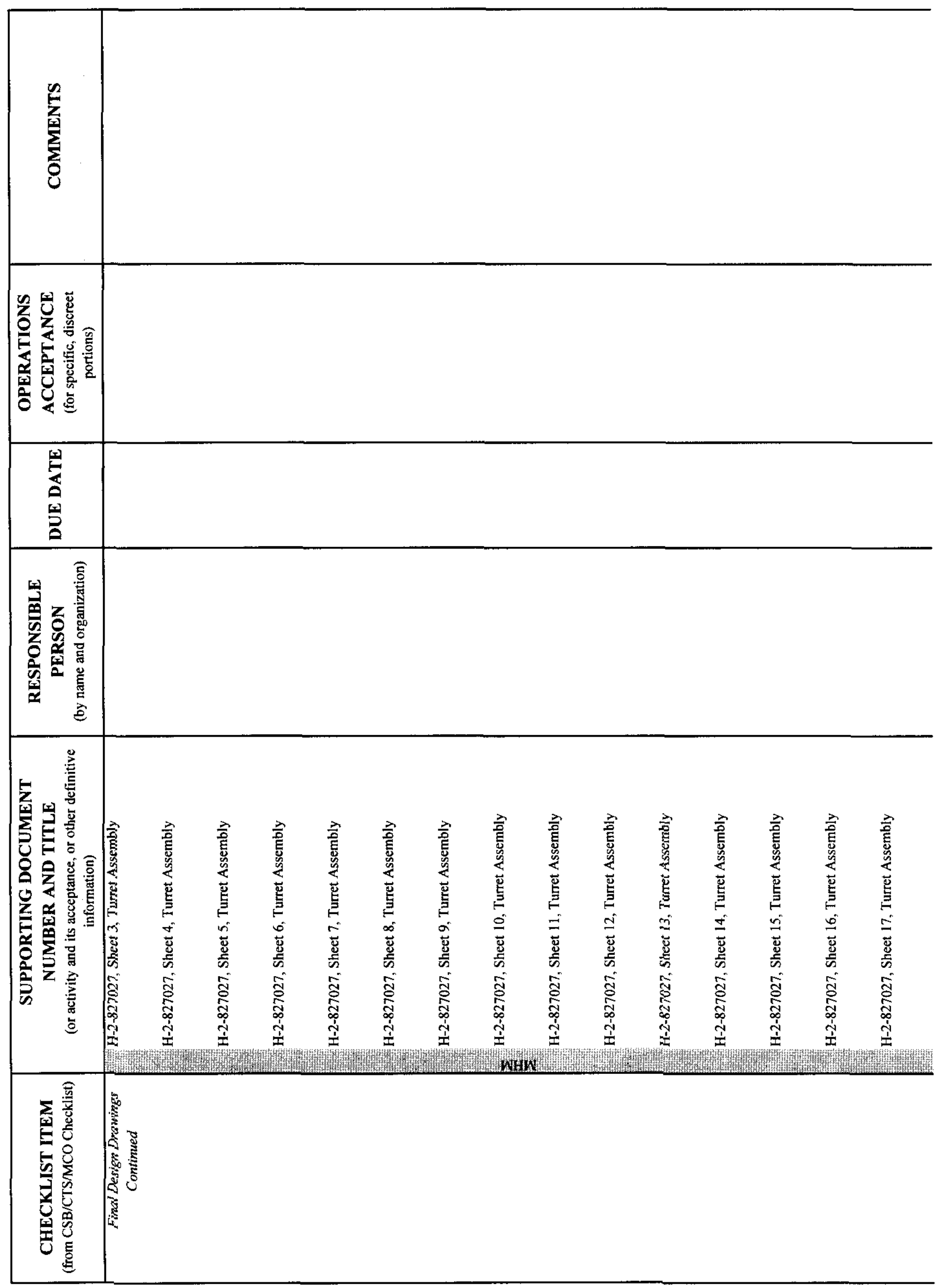


SNF-6253 REV 0

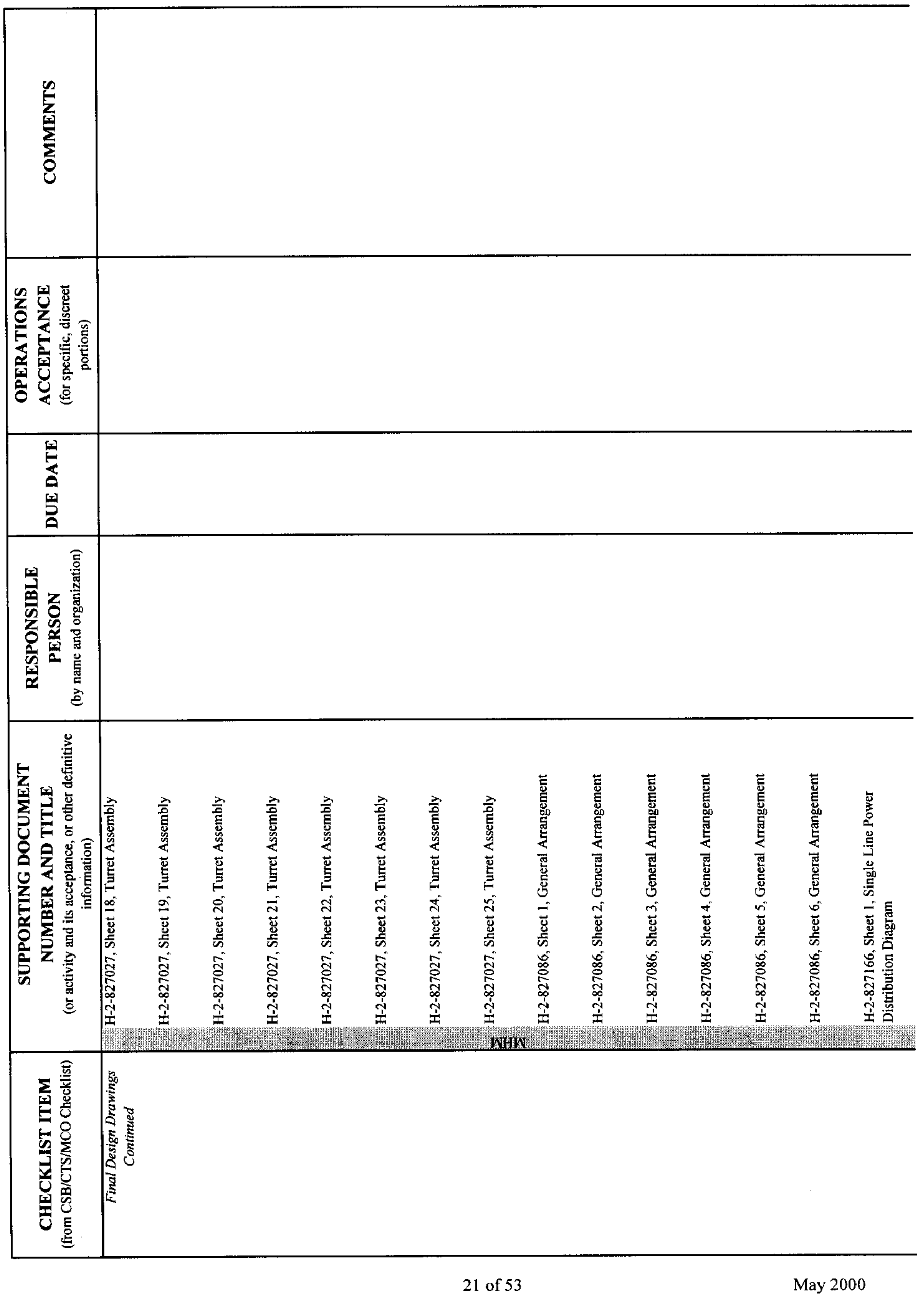




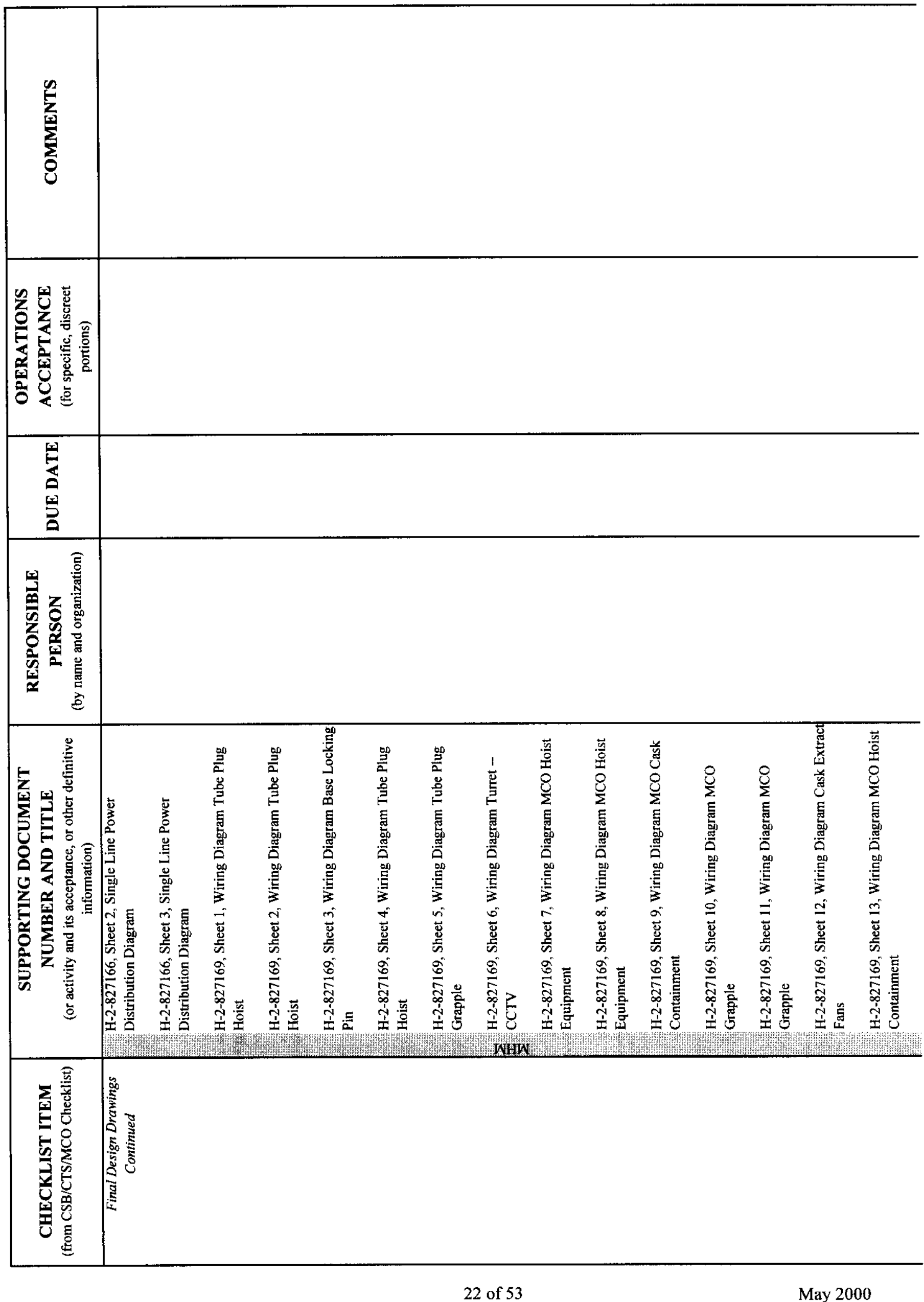




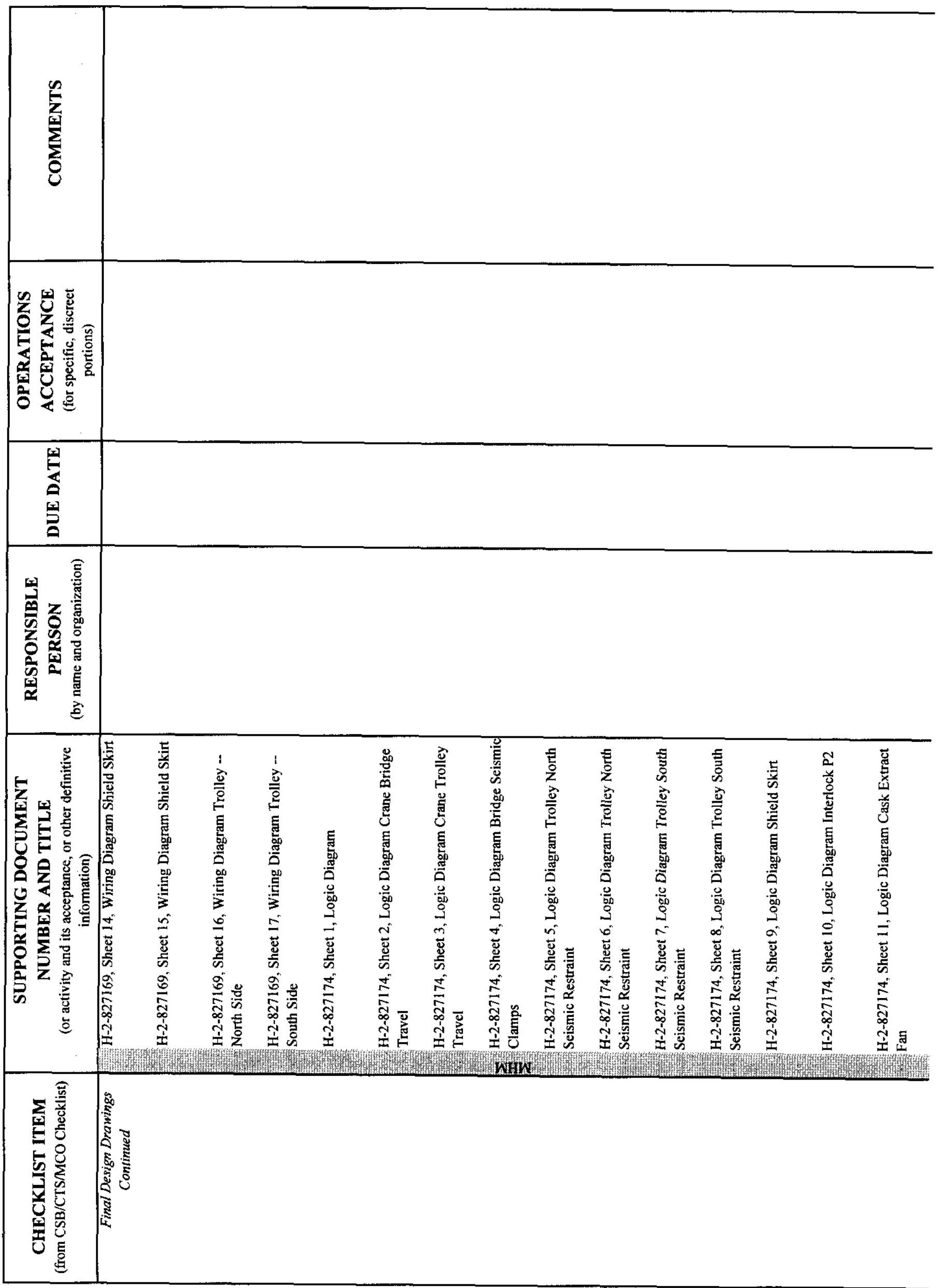


SNF-6253 REV 0

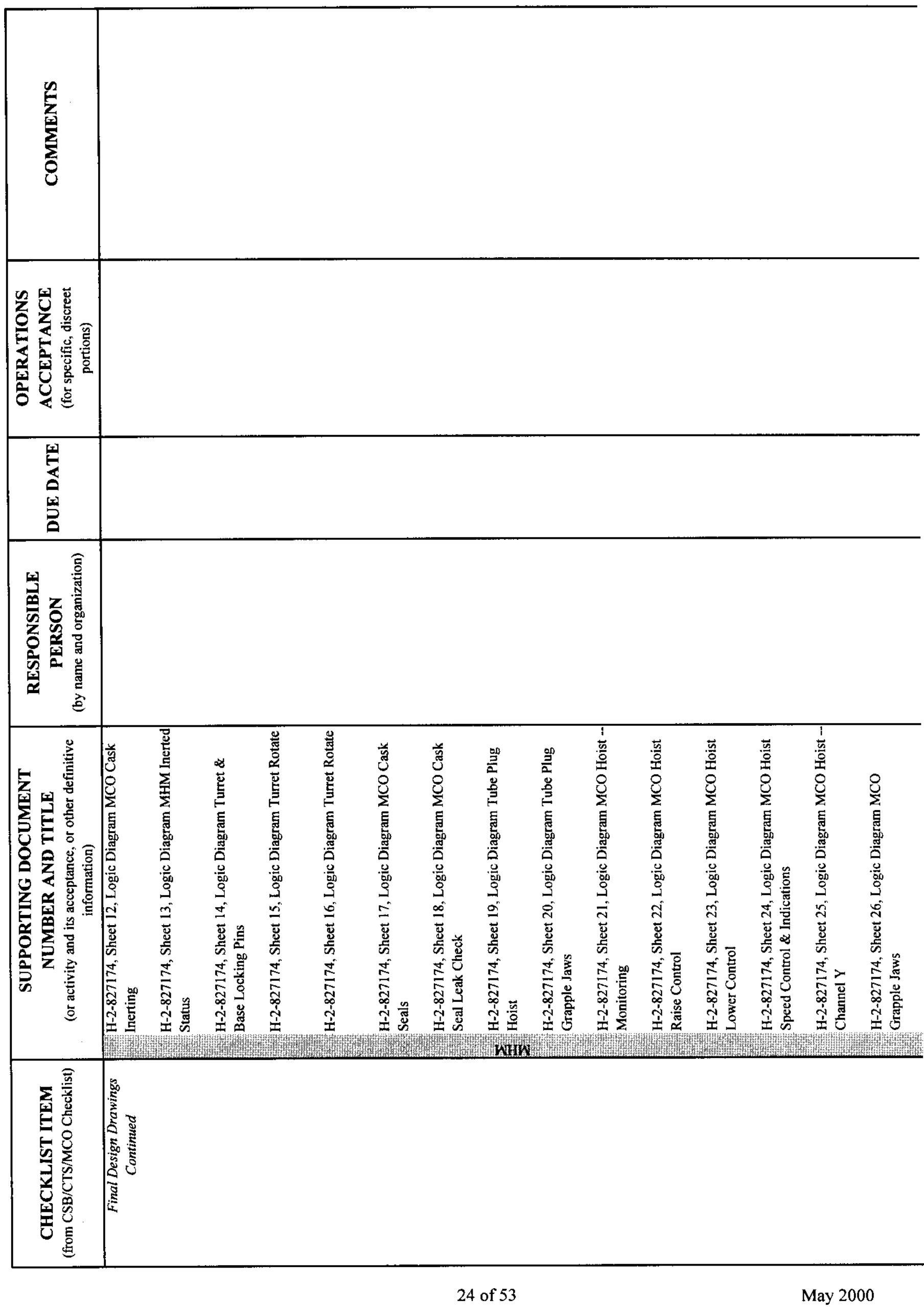




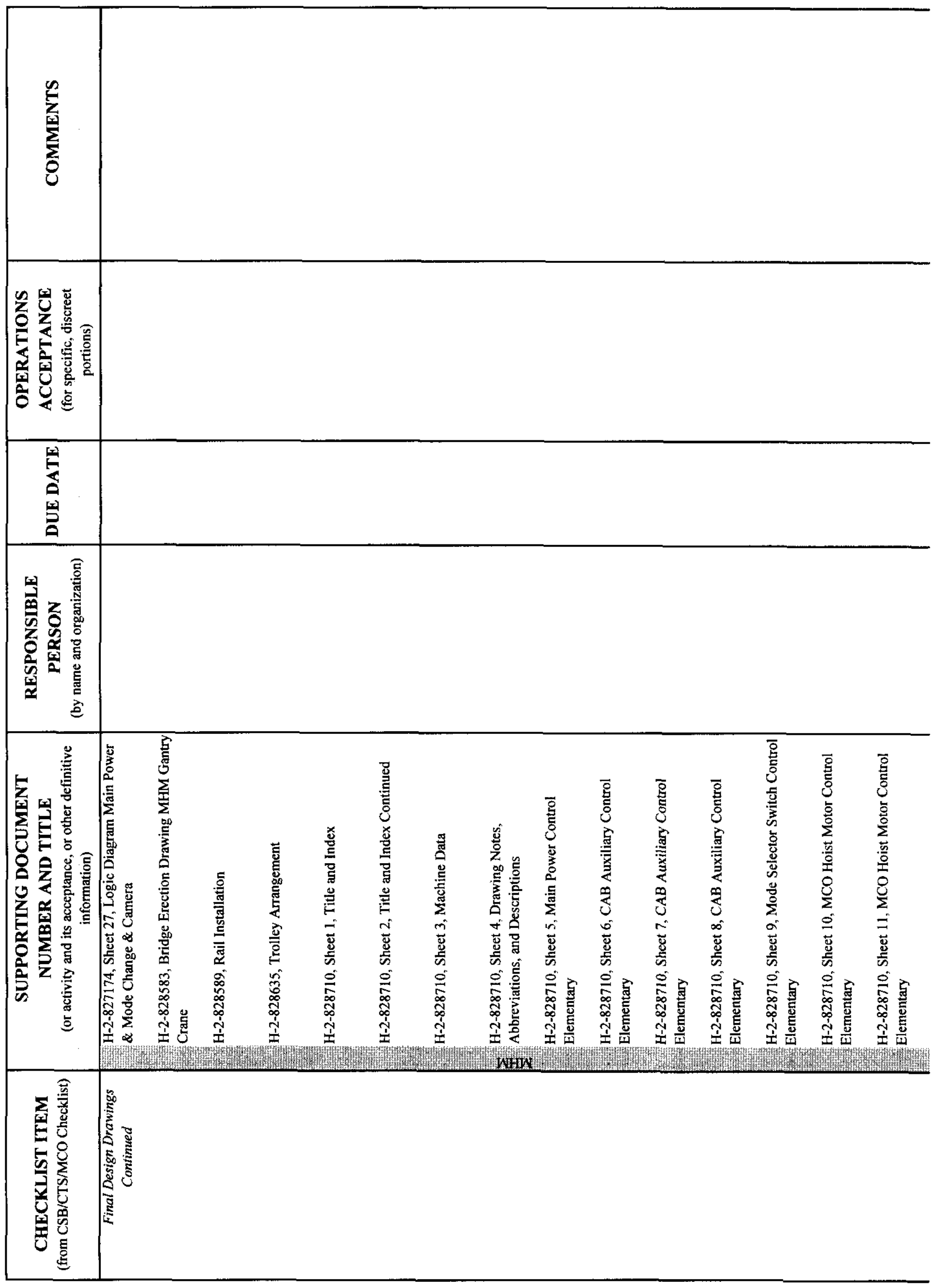


SNF-6253 REV 0

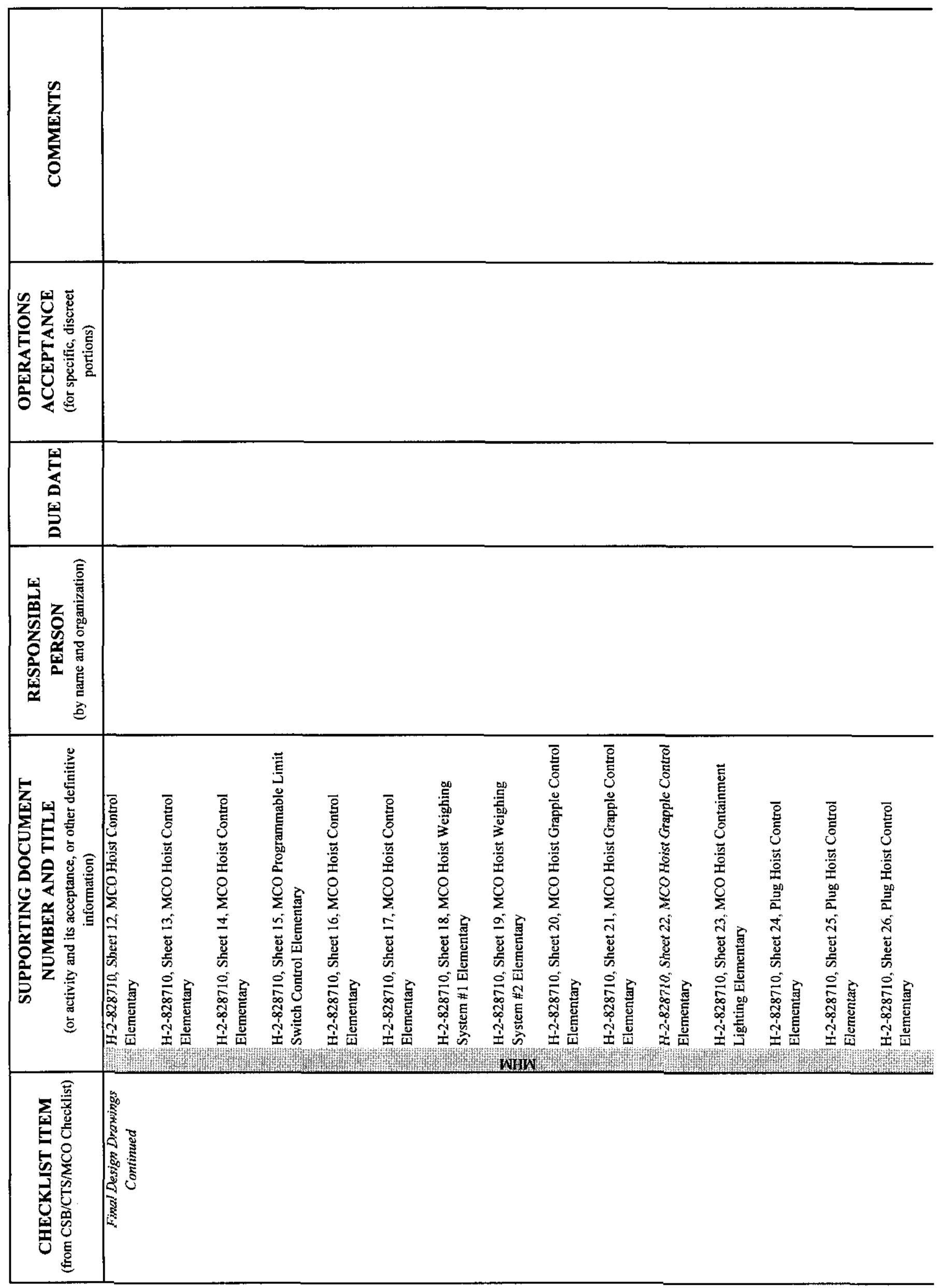


SNF-6253 REV 0

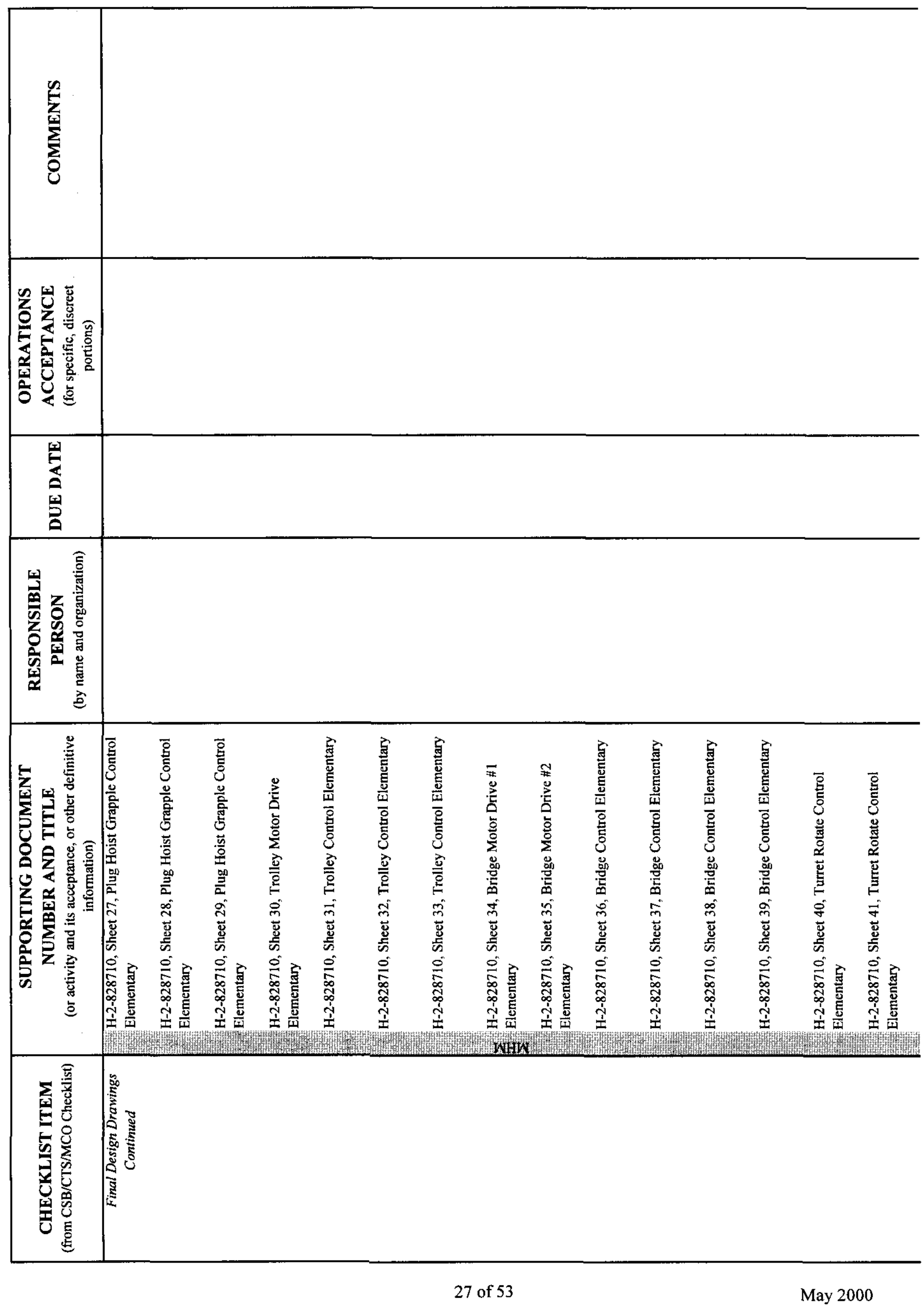


SNF-6253 REV 0

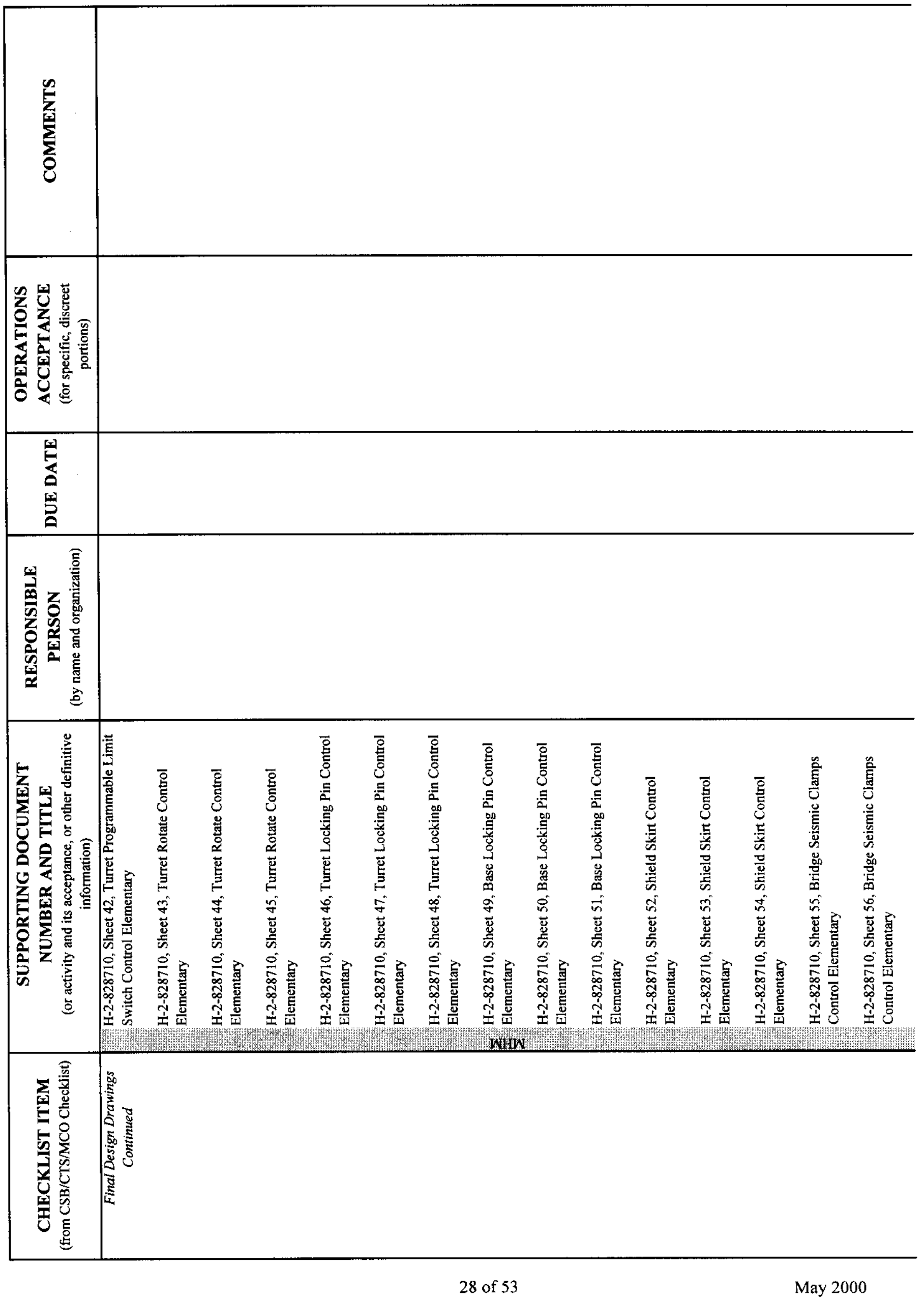




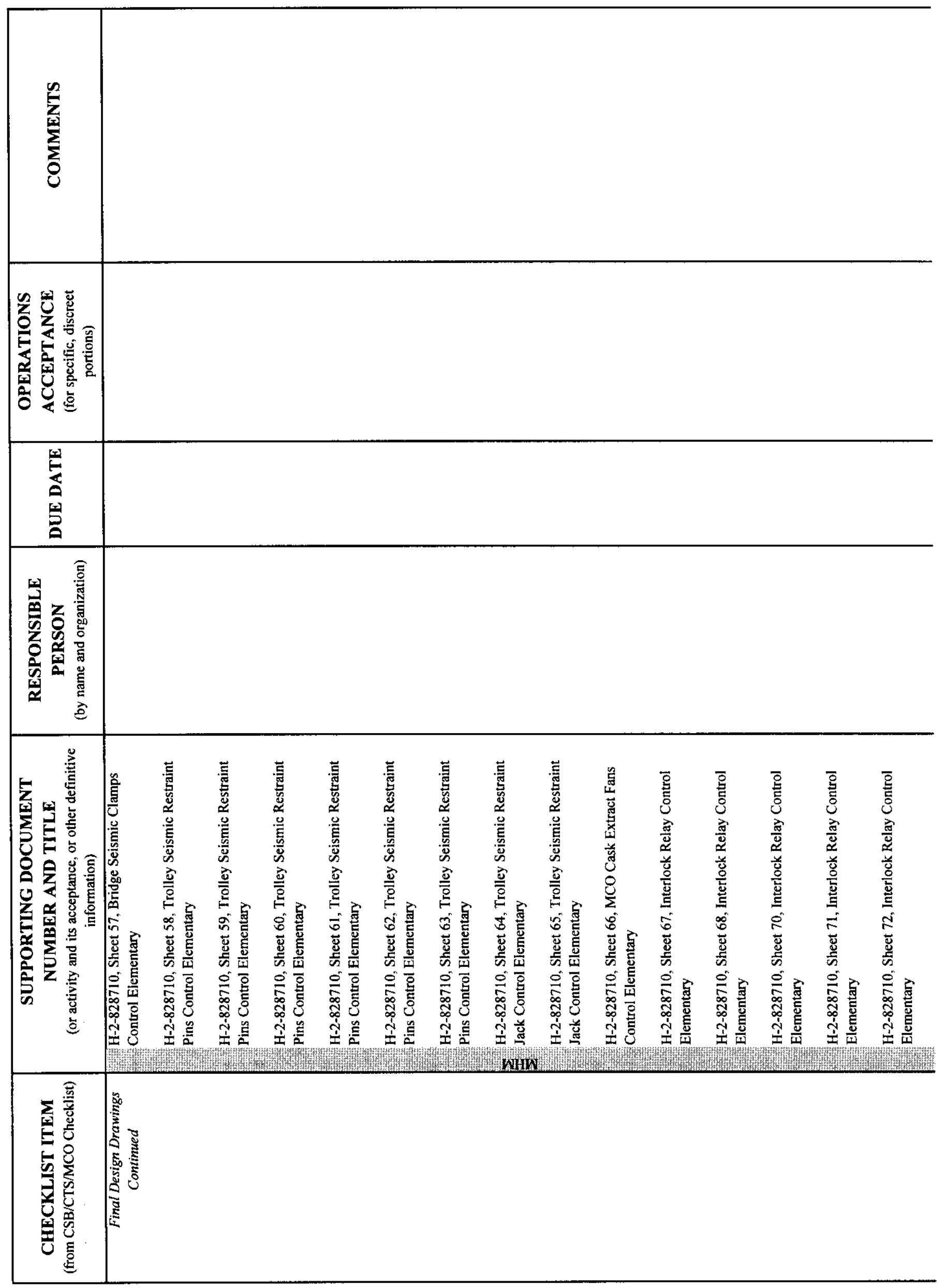




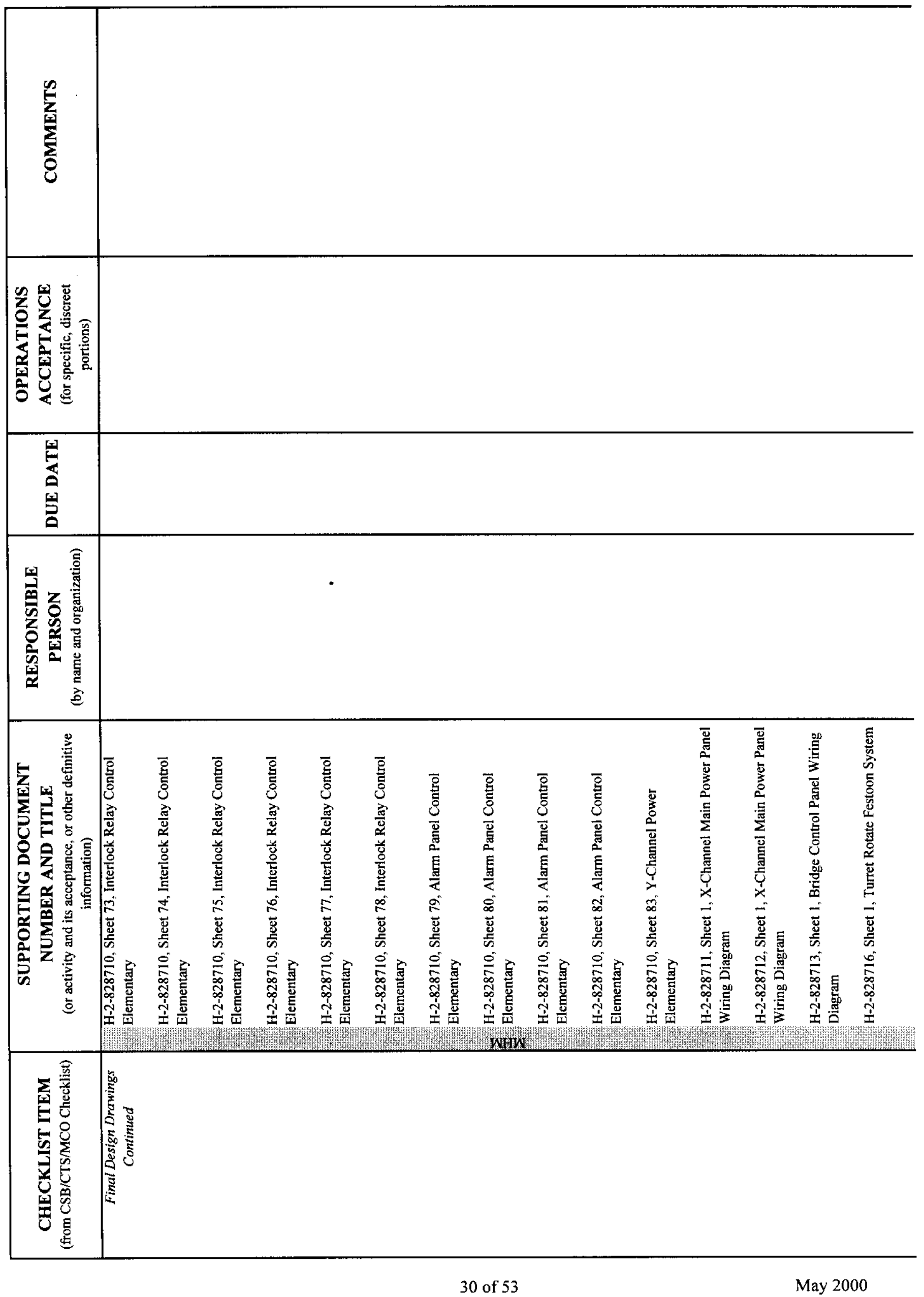


SNF-6253 REV 0

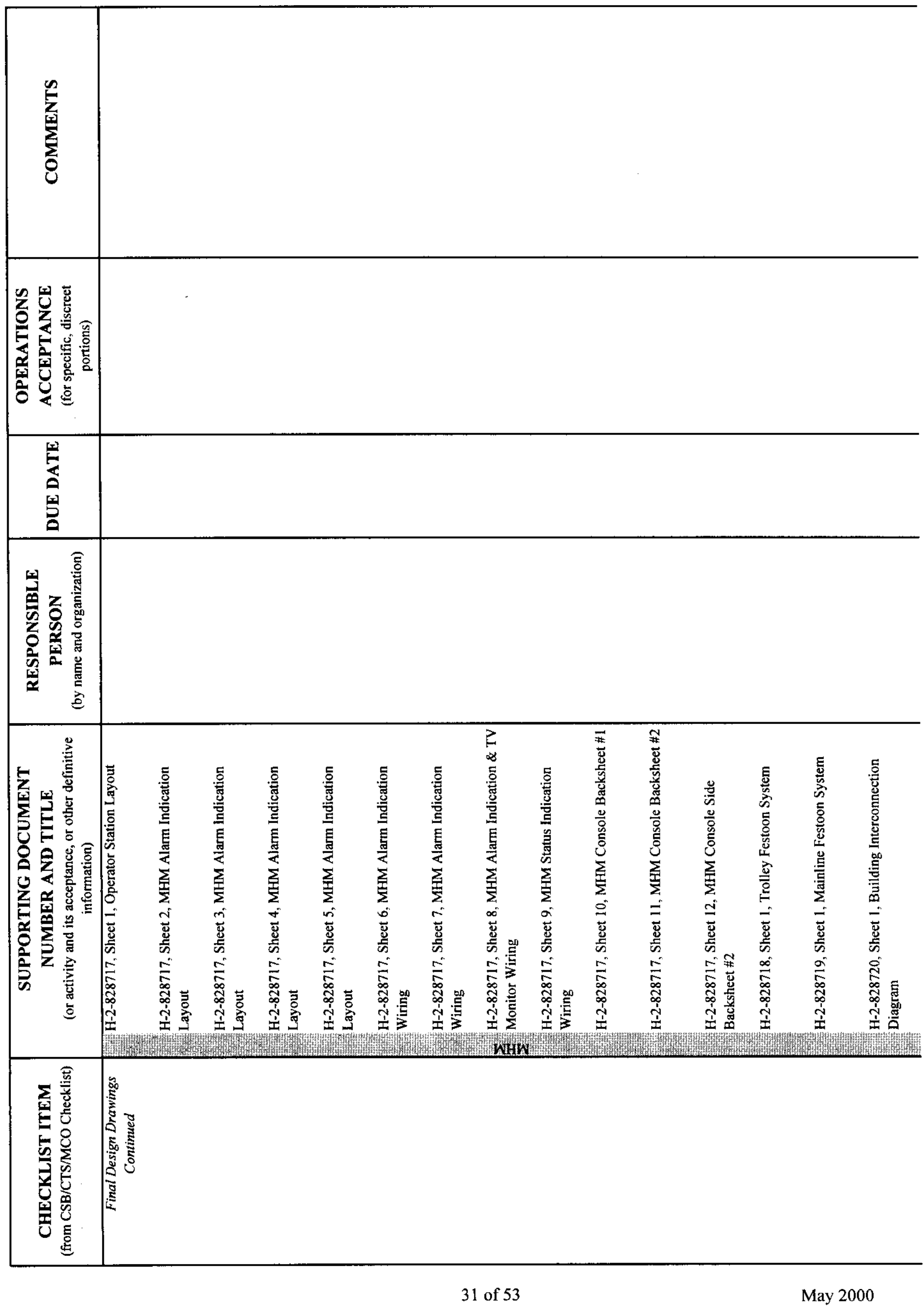


SNF-6253 REV 0

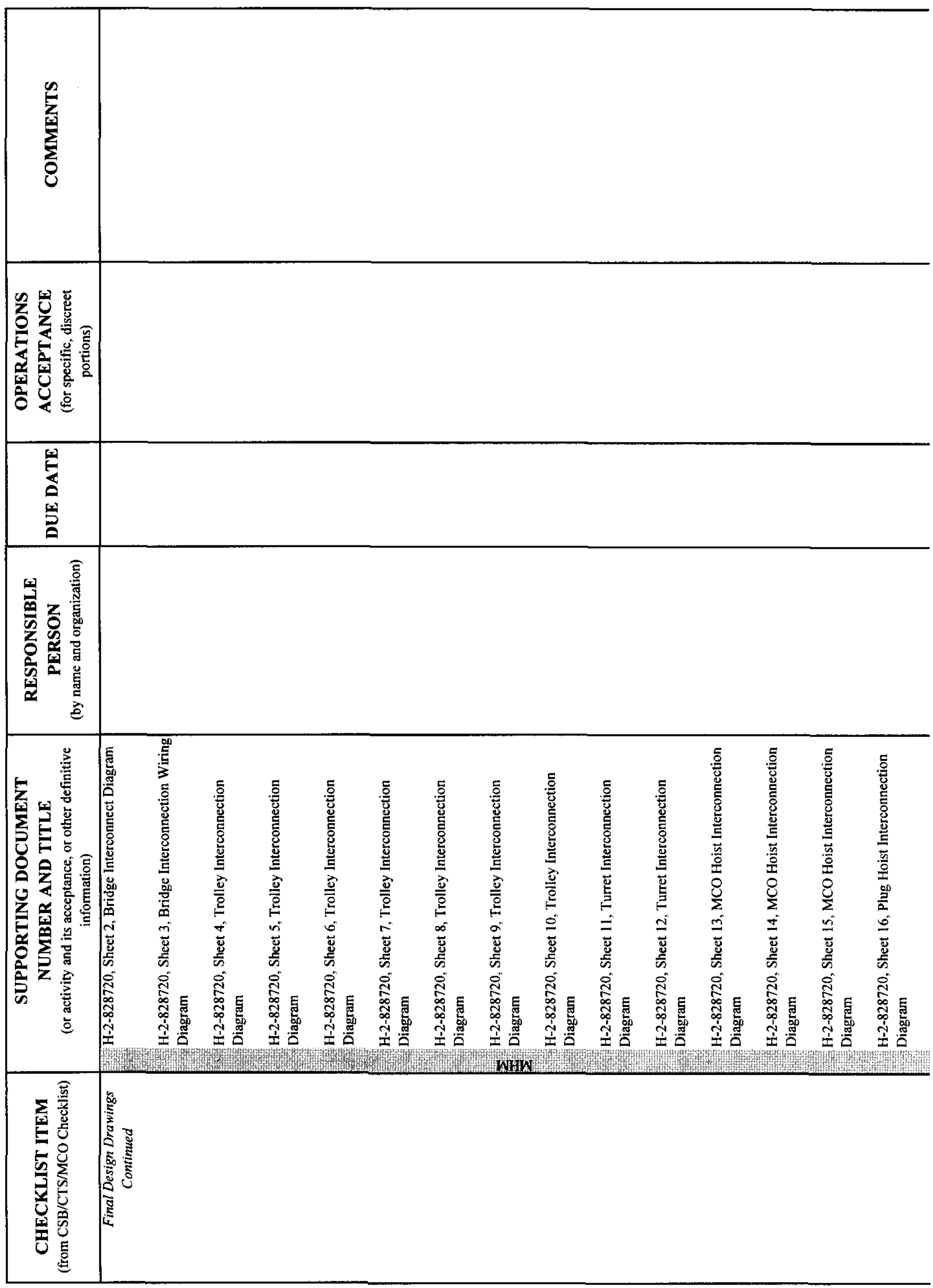




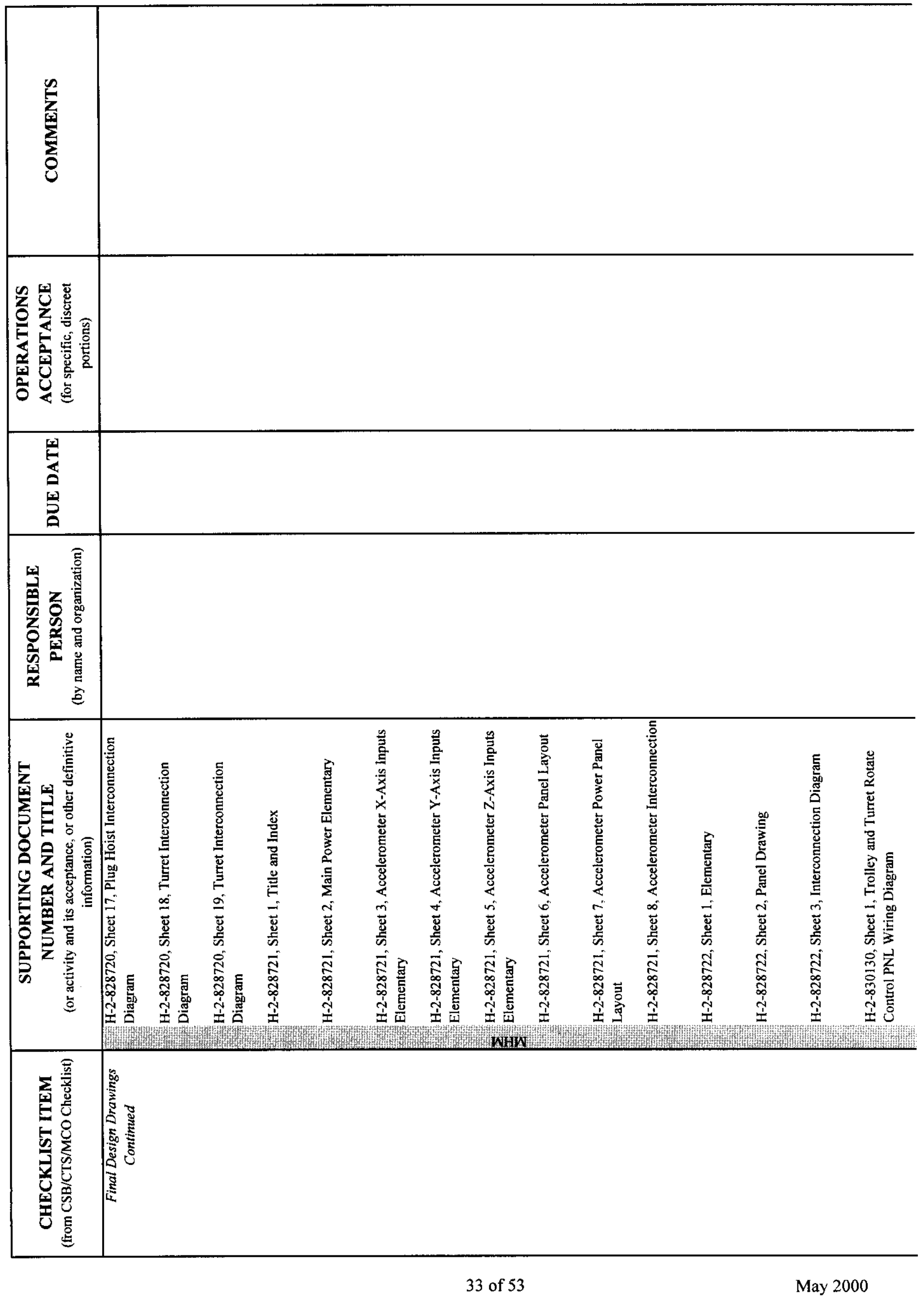




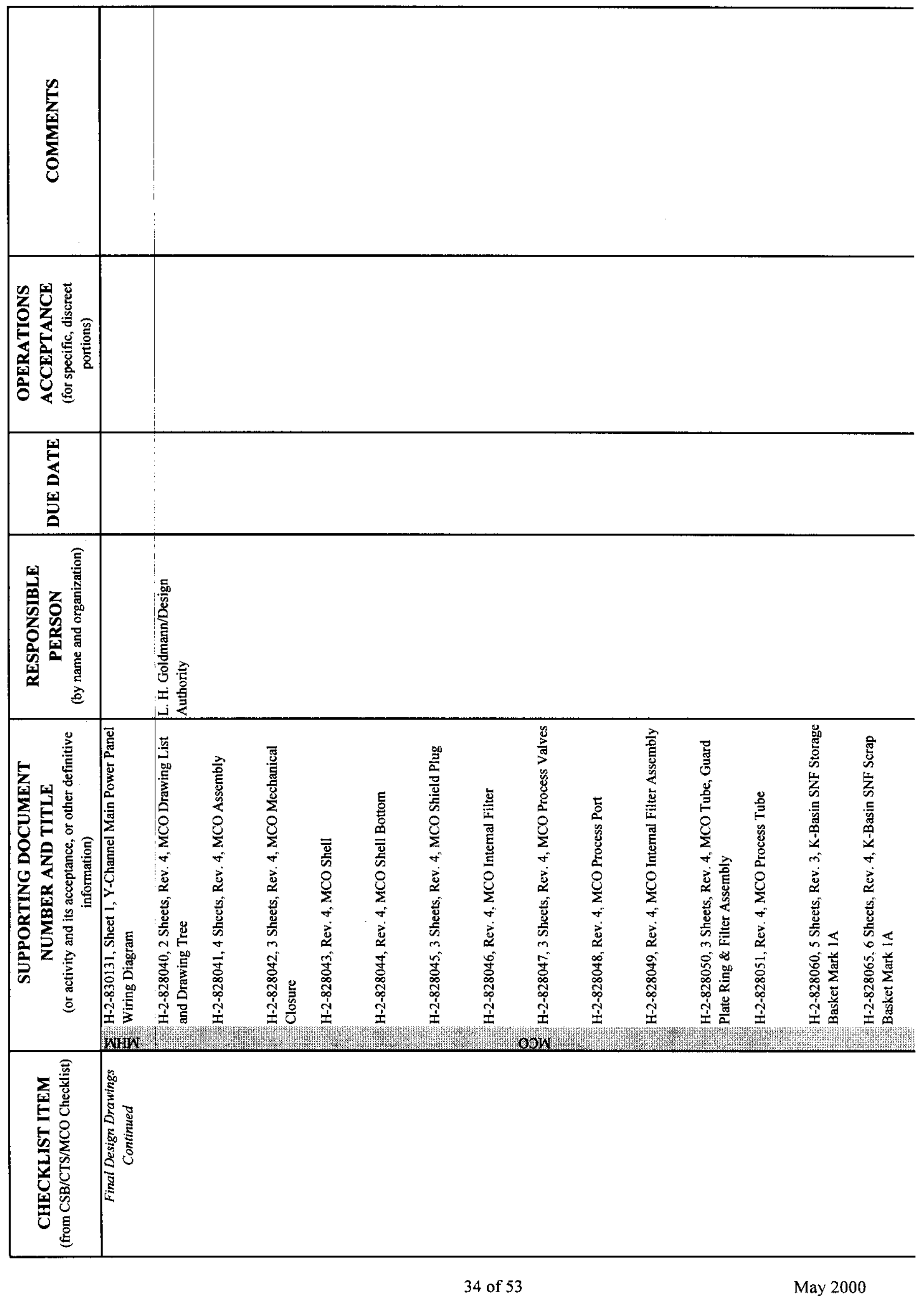


SNF-6253 REV 0

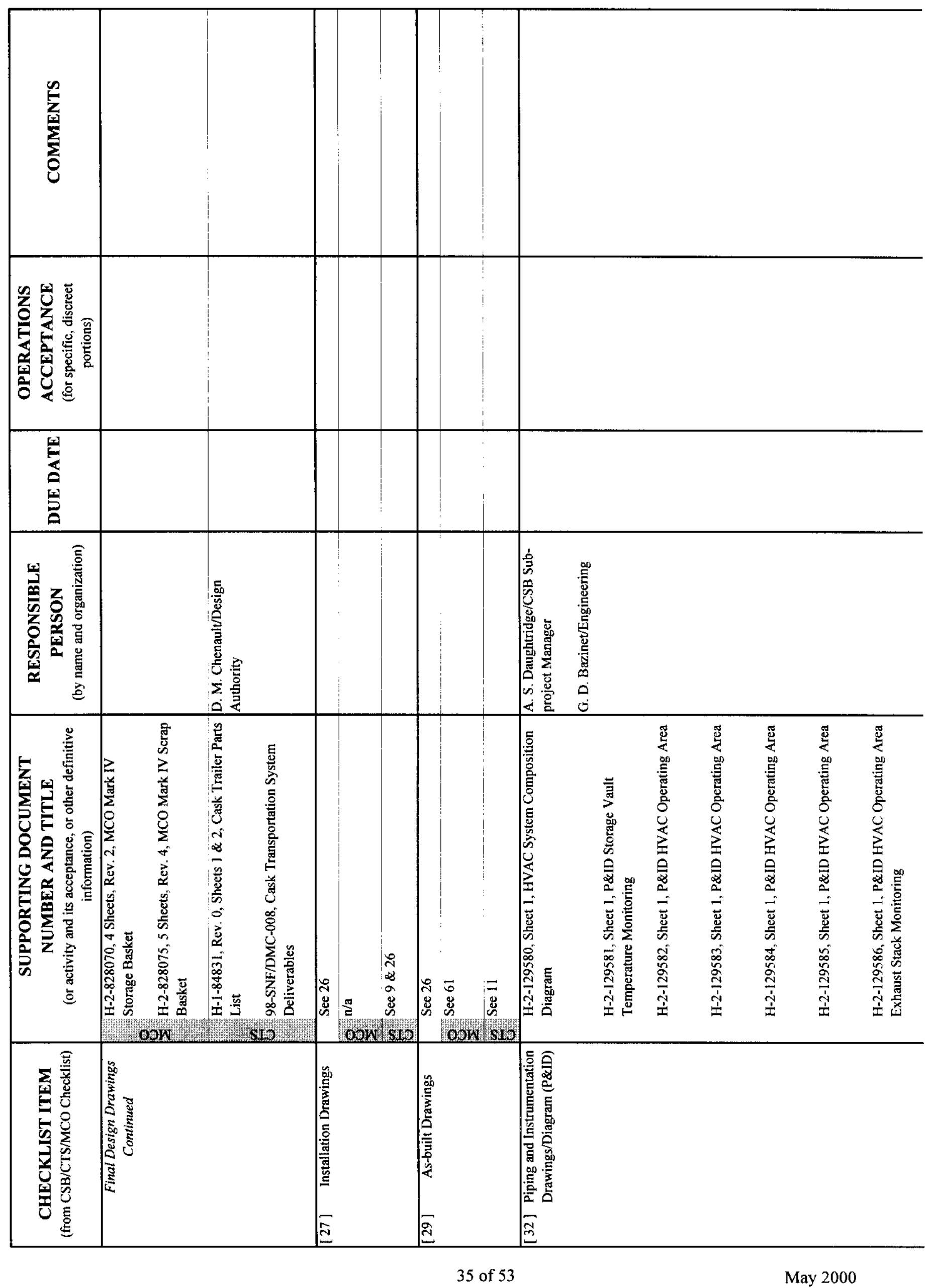


SNF-6253 REV 0

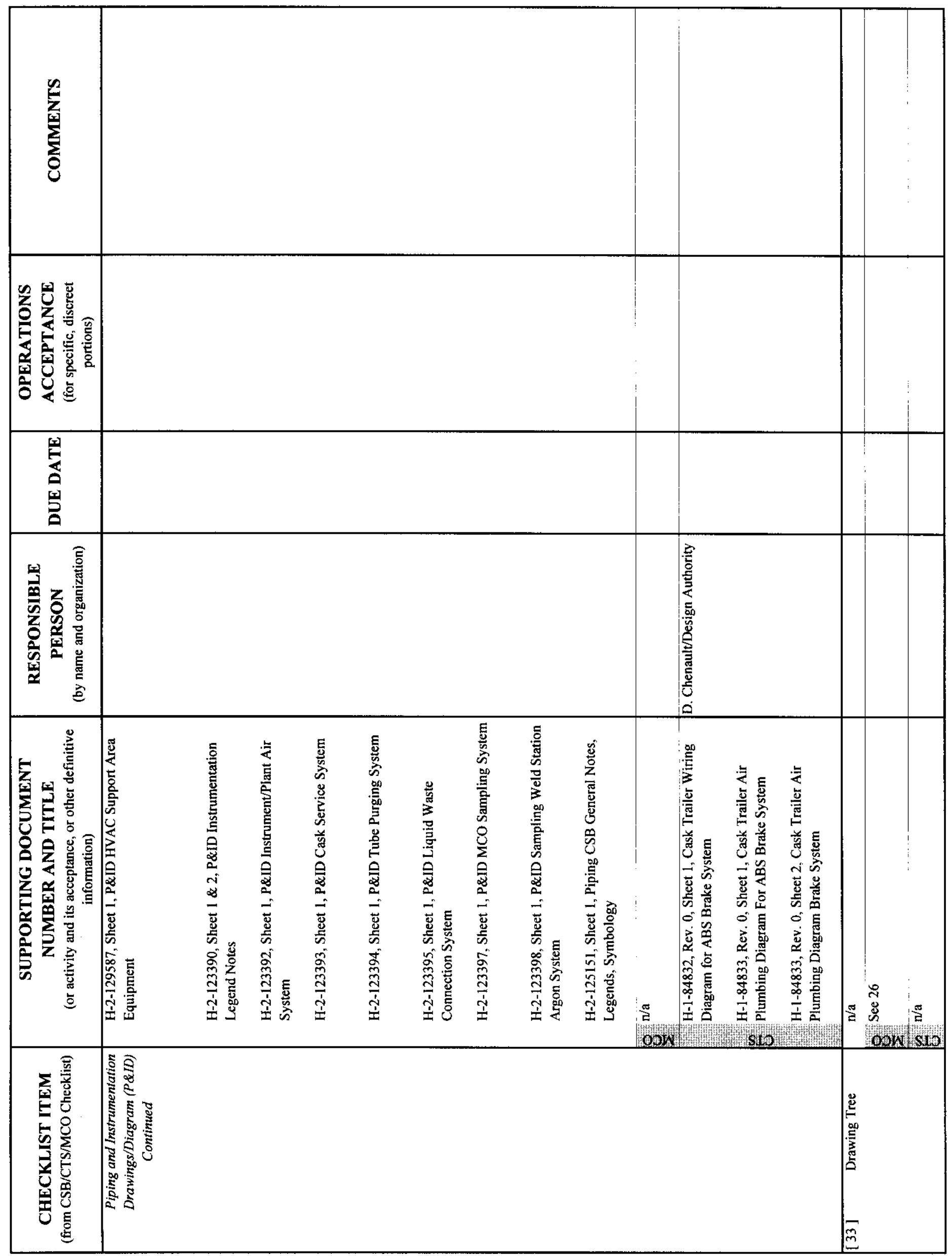


SNF-6253 REV 0

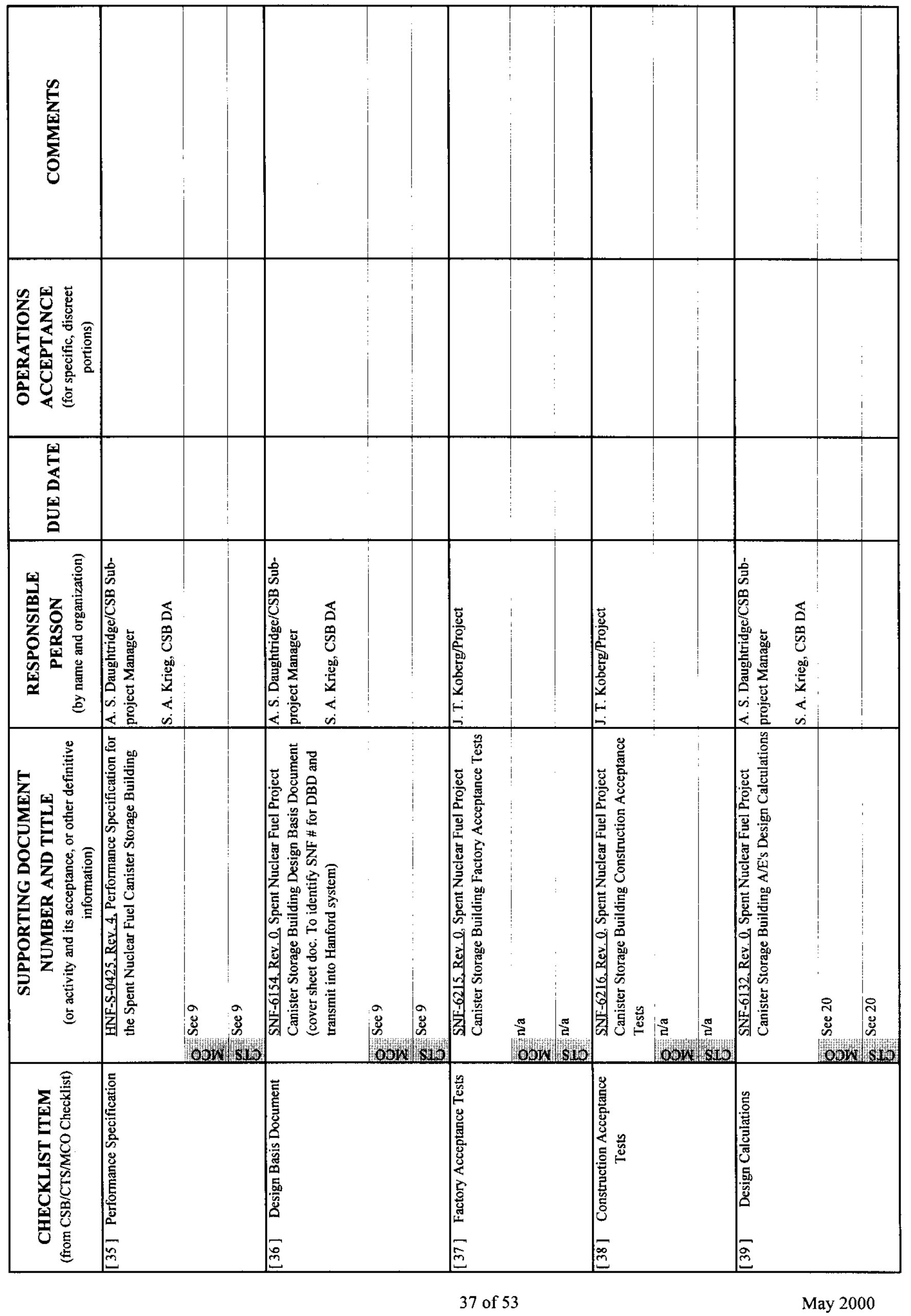


SNF-6253 REV 0

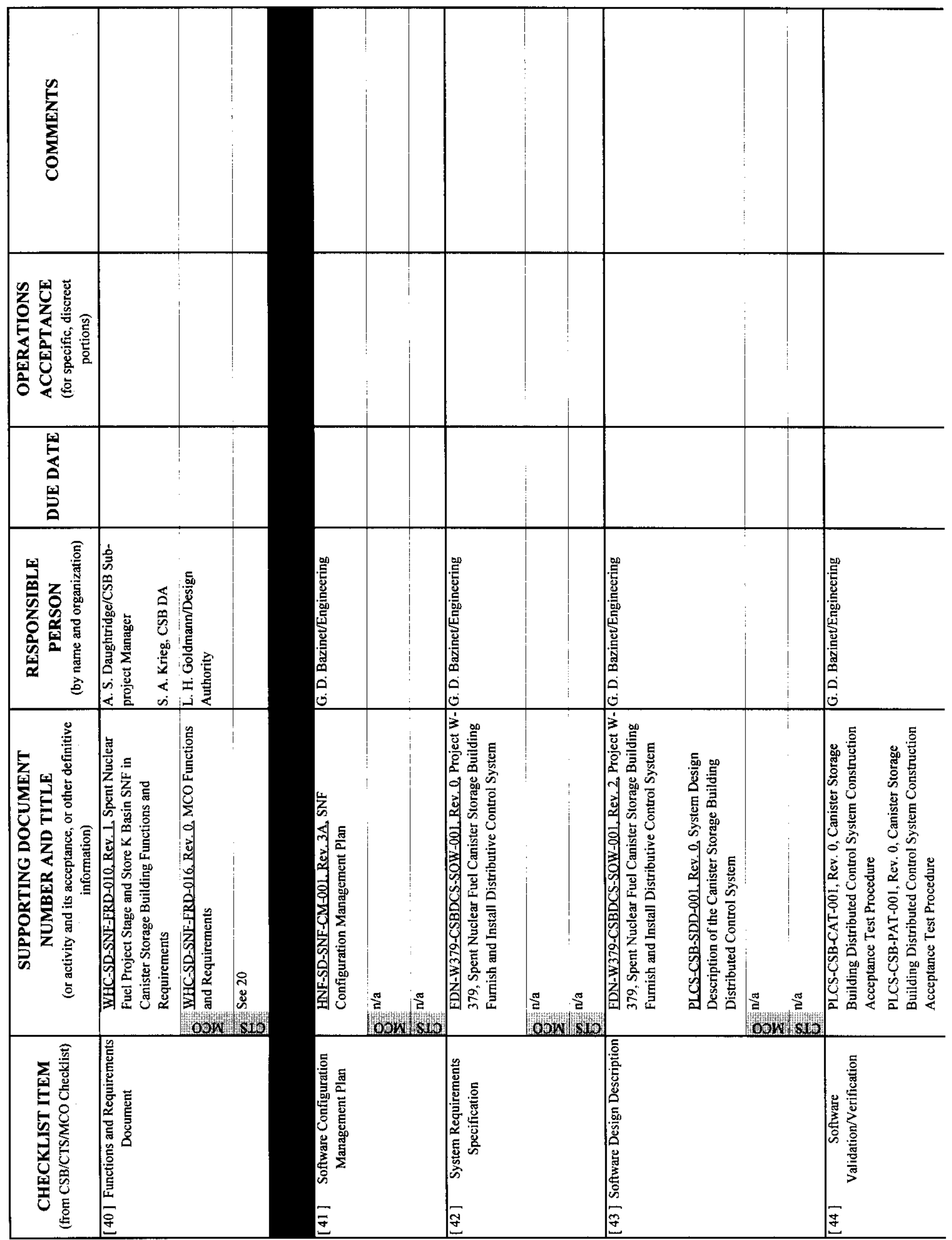




$$
\mid
$$


SNF-6253 REV 0

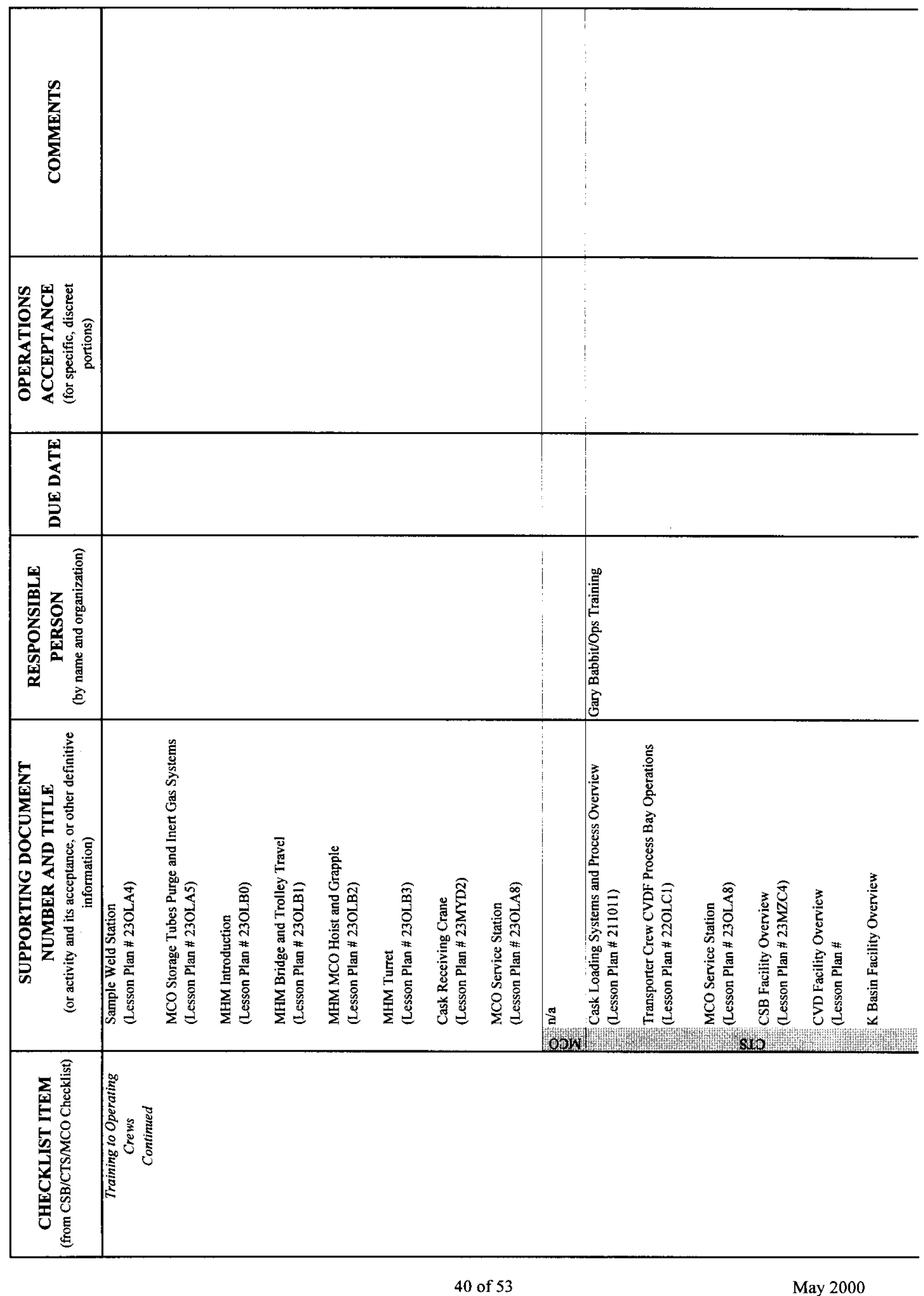


SNF-6253 REV 0

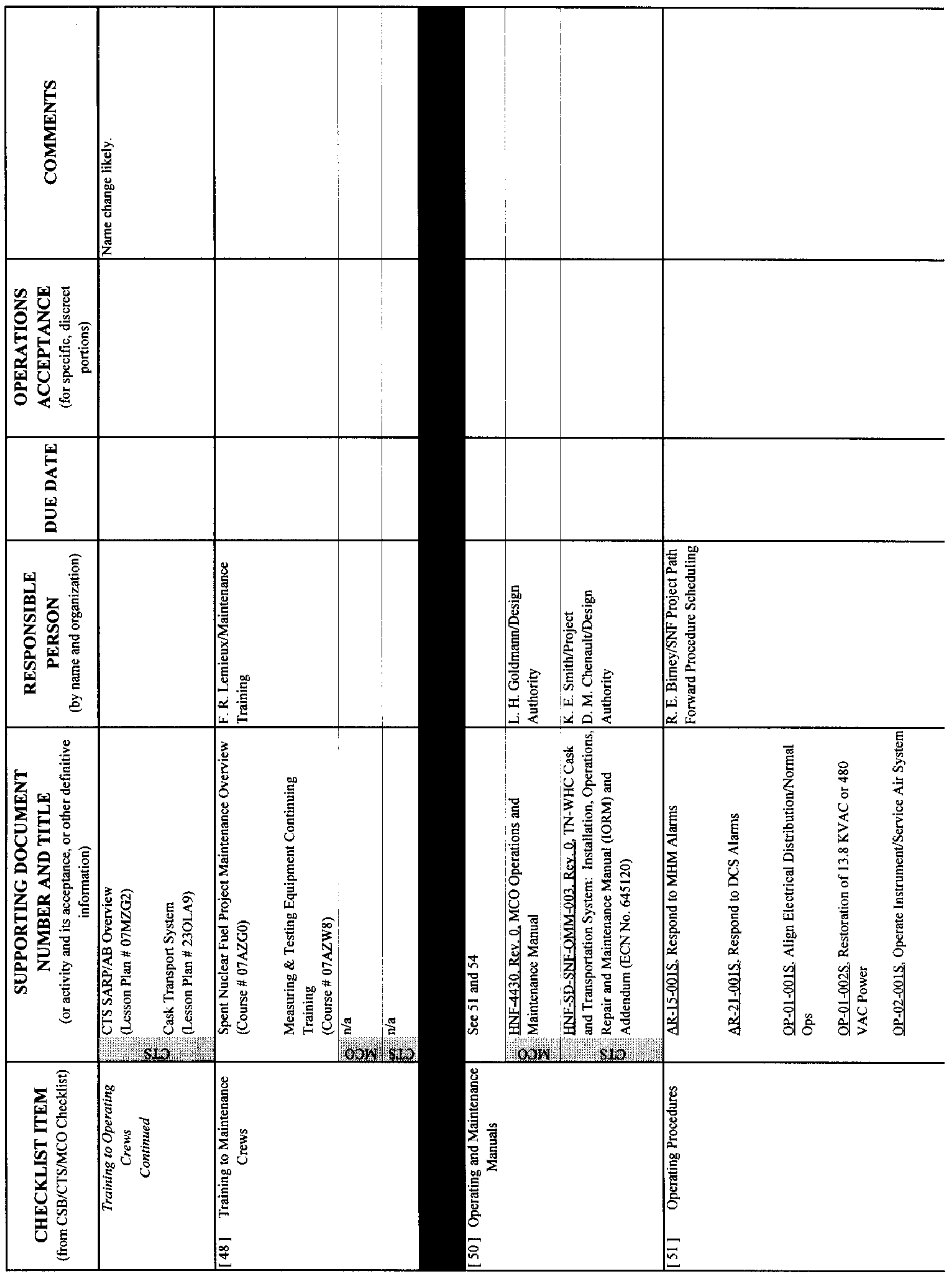


SNF-6253 REV 0

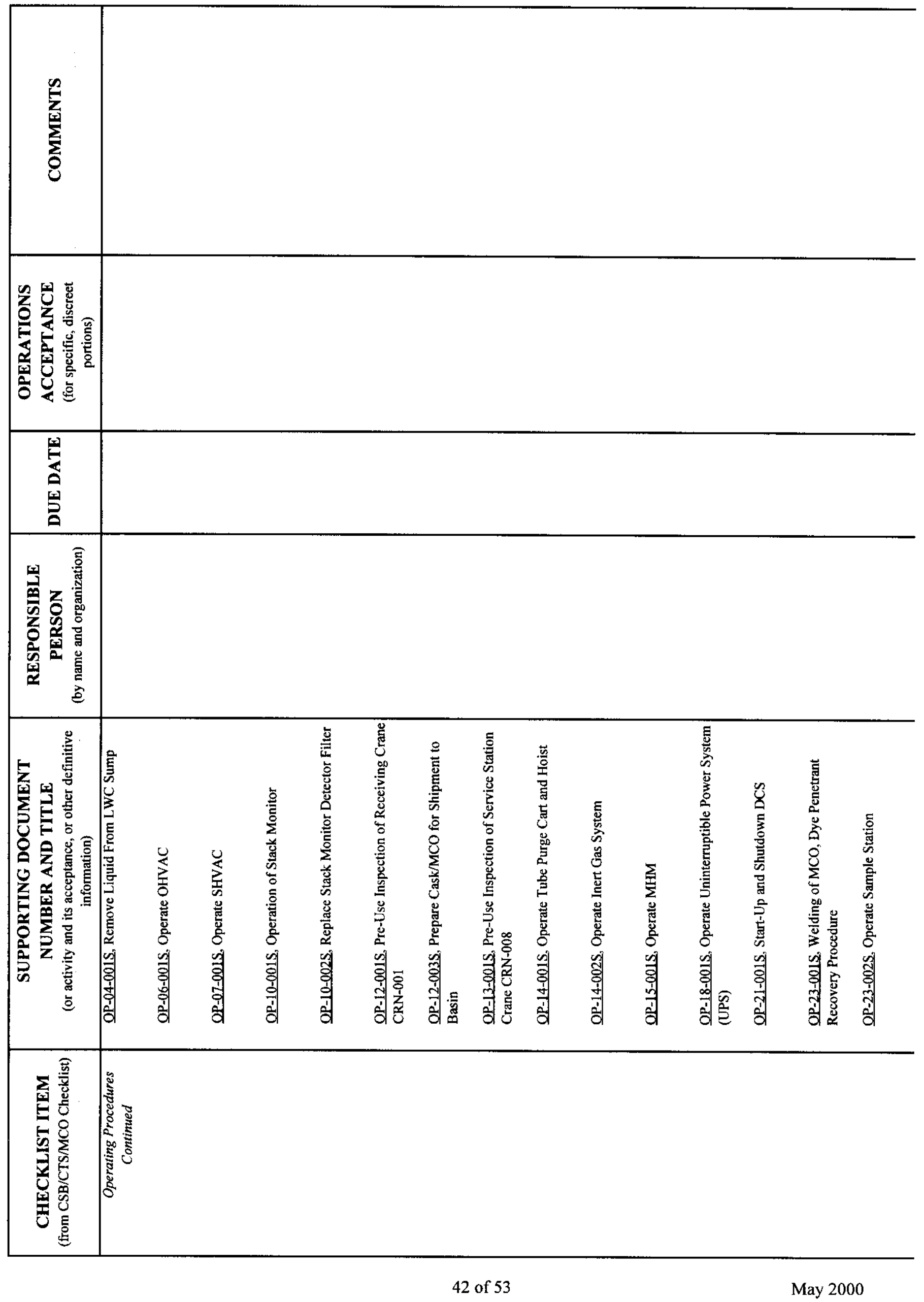


SNF-6253 REV 0

\begin{tabular}{|c|c|c|c|c|c|c|c|c|c|c|c|c|c|c|c|c|}
\hline$\sum_{0}^{\infty}$ & & & 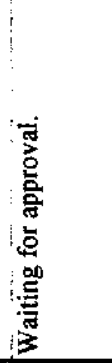 & & & & & & & & & & & & & \\
\hline 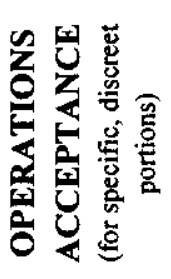 & & & & & & & & & & & & & & & & \\
\hline $\begin{array}{l}\text { 됩 } \\
\text { 品 } \\
\text { 될 } \\
0\end{array}$ & & & & & & & & & & & & & & & & \\
\hline 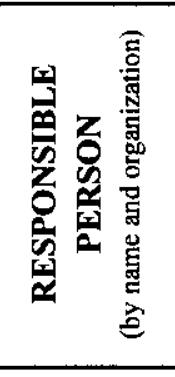 & & $\begin{array}{l}: \\
\vdots \\
\vdots \\
\vdots\end{array}$ & 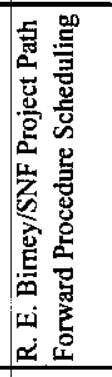 & & : & 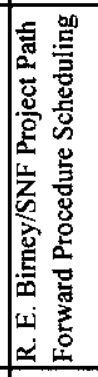 & & & & & & & & & & \\
\hline 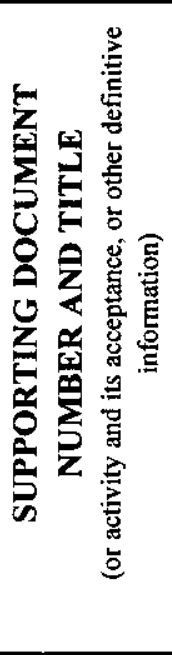 & 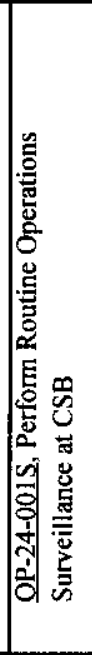 & oax & 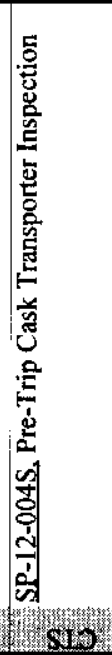 & 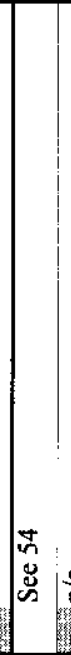 & 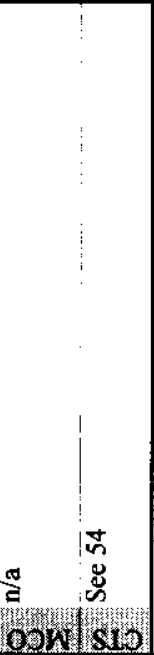 & 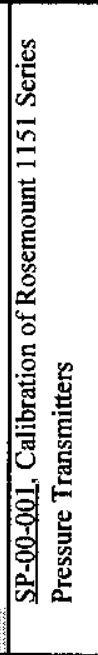 & 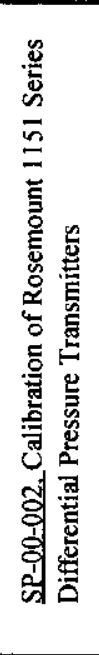 & 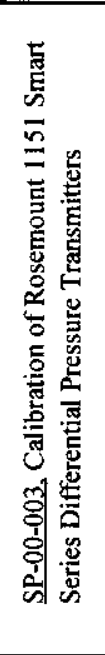 & 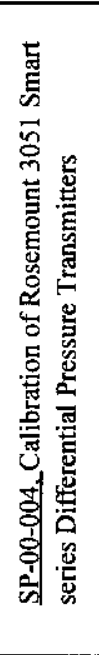 & 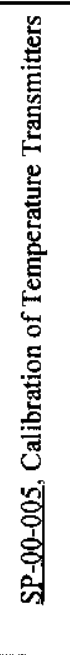 & 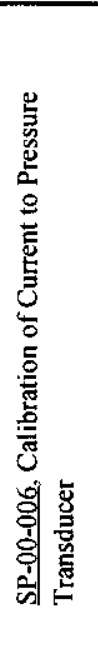 & 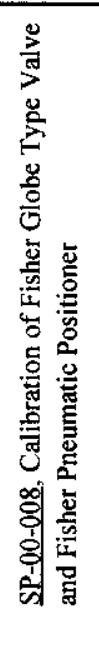 & 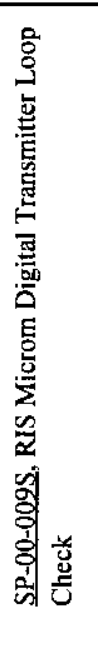 & 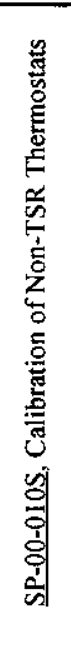 & 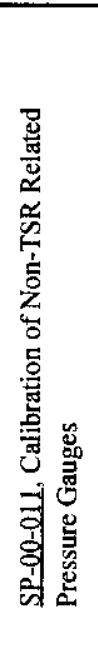 & 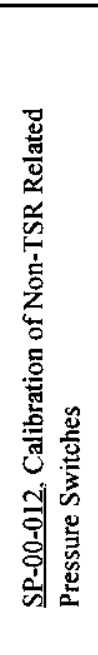 \\
\hline 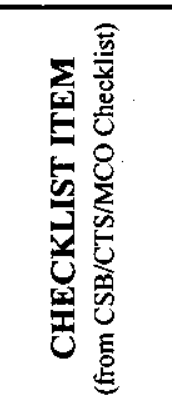 & 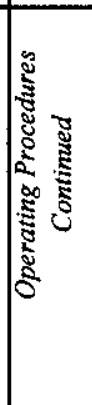 & & & 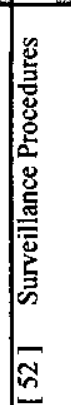 & & 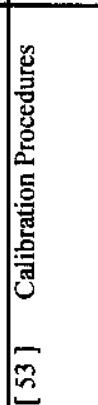 & & & & & & & & & & \\
\hline
\end{tabular}


SNF-6253 REV 0

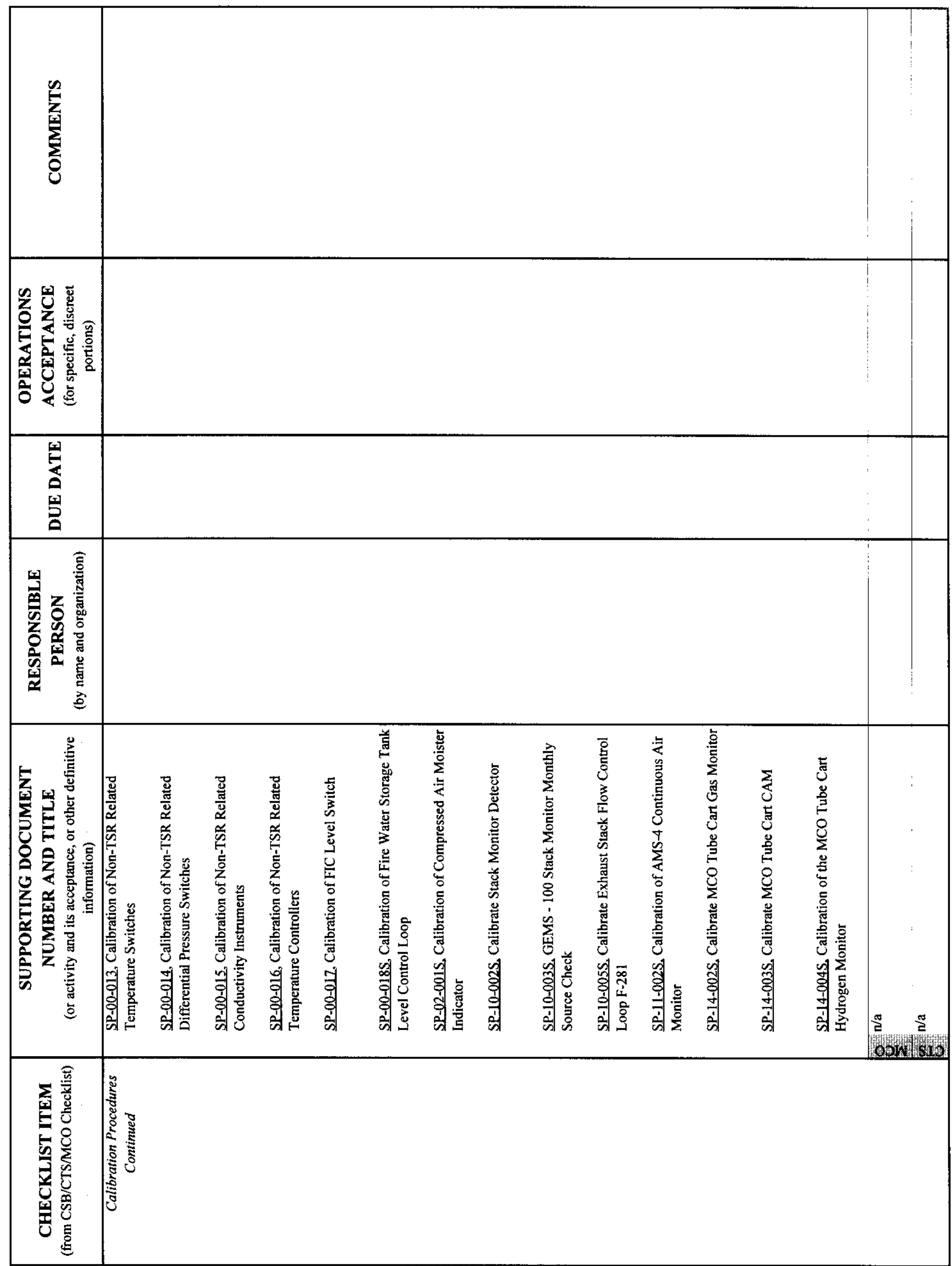


SNF-6253 REV 0

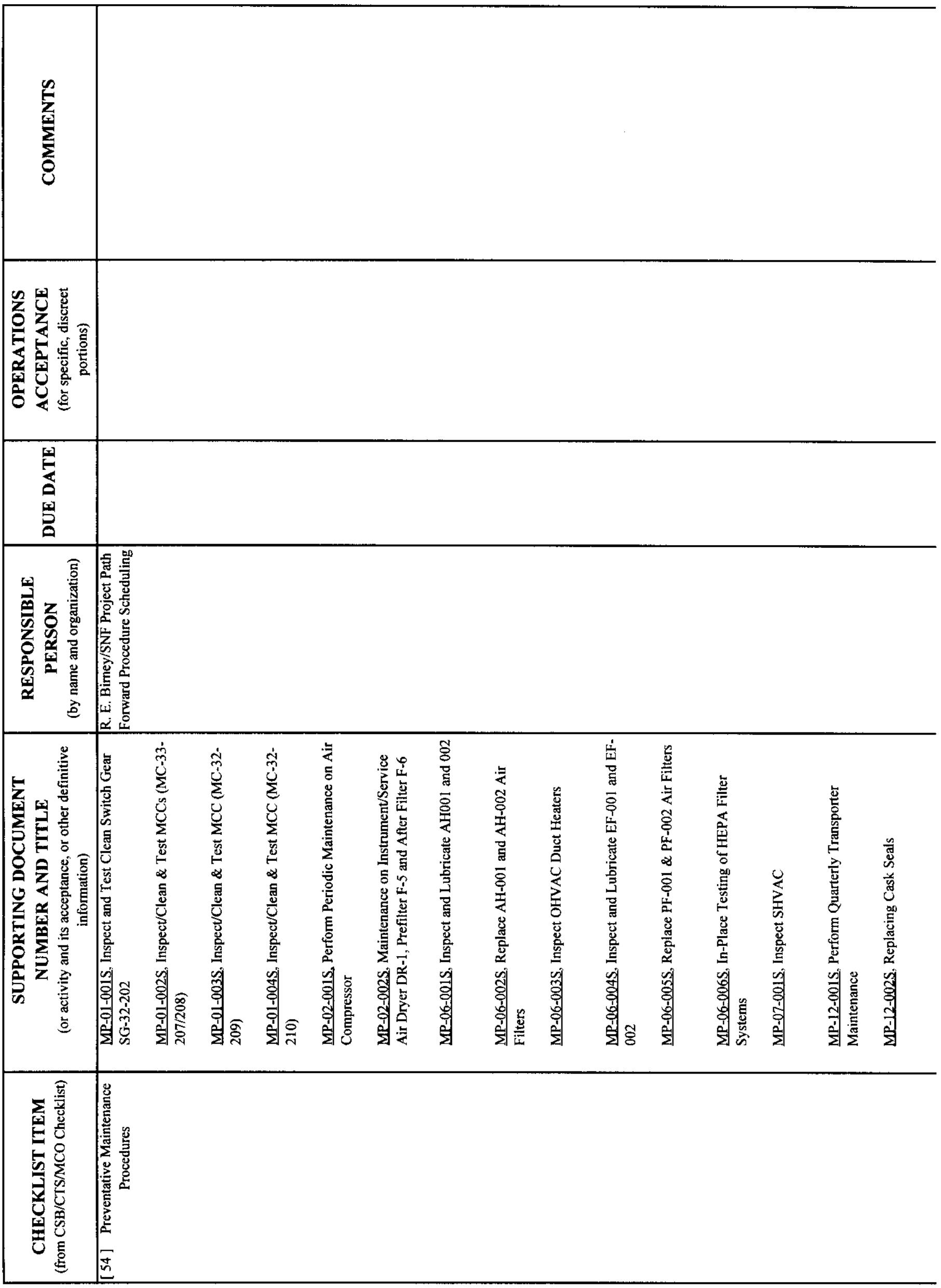


SNF-6253 REV 0

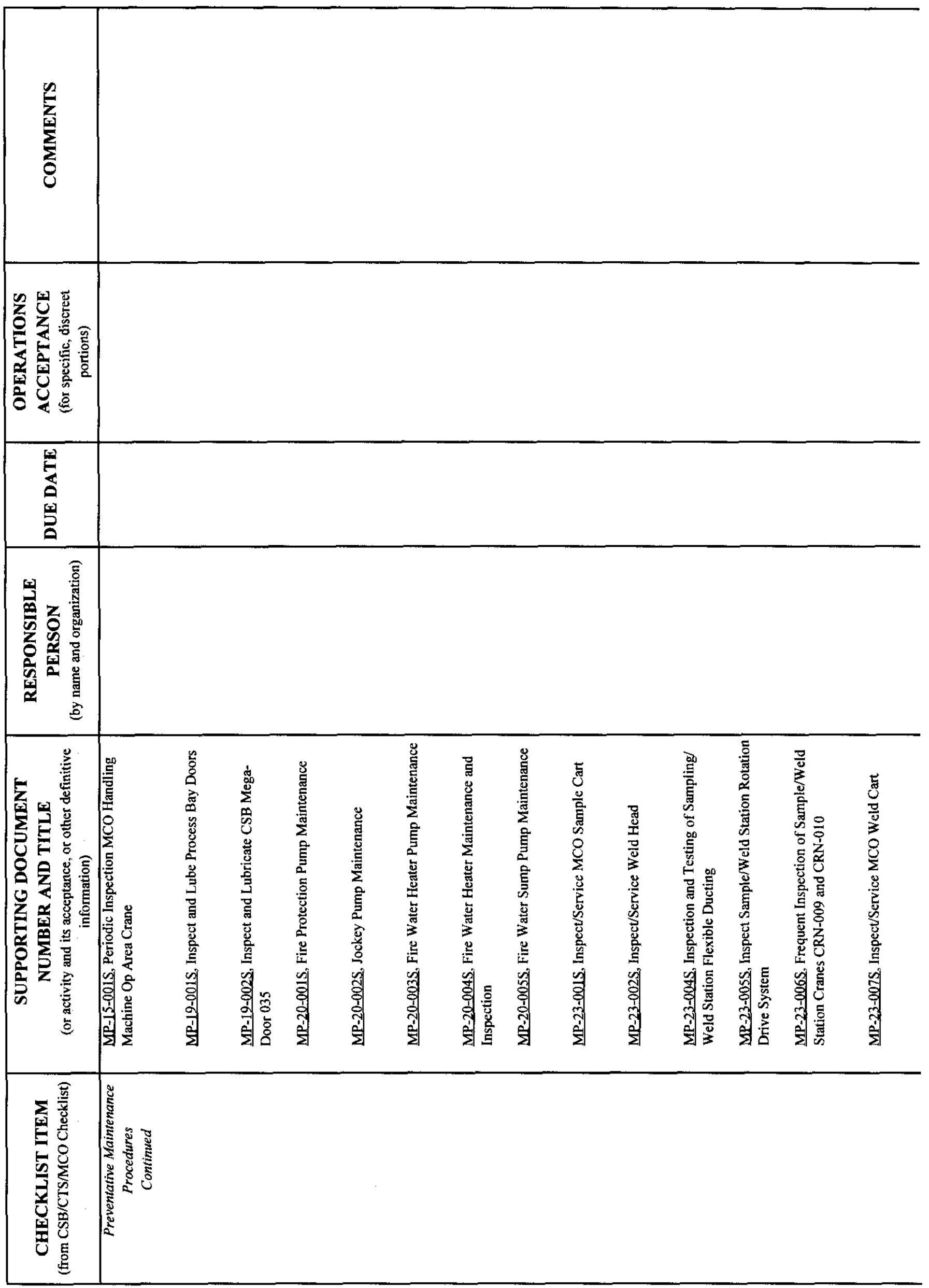


SNF-6253 REV 0

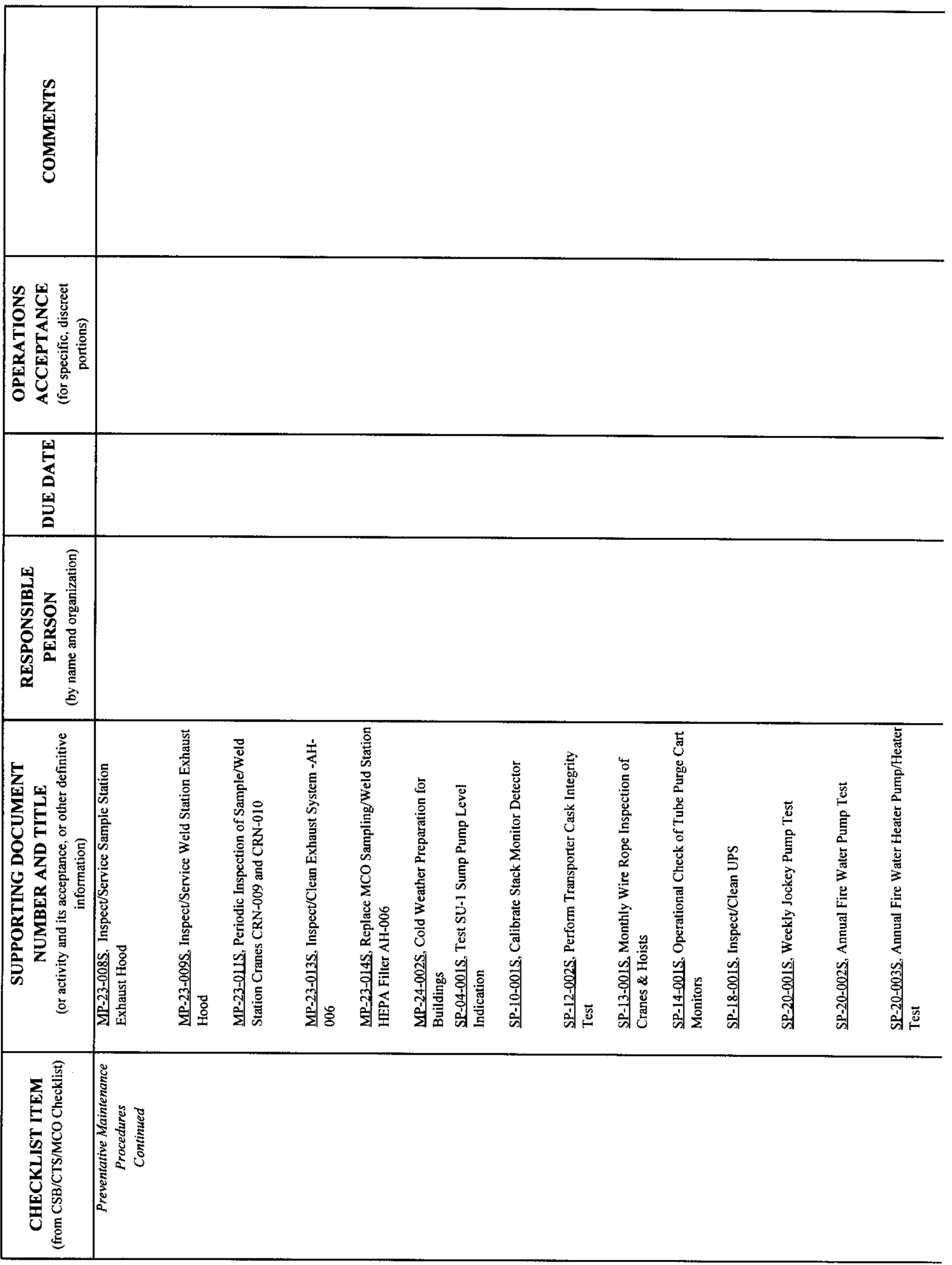


SNF-6253 REV 0

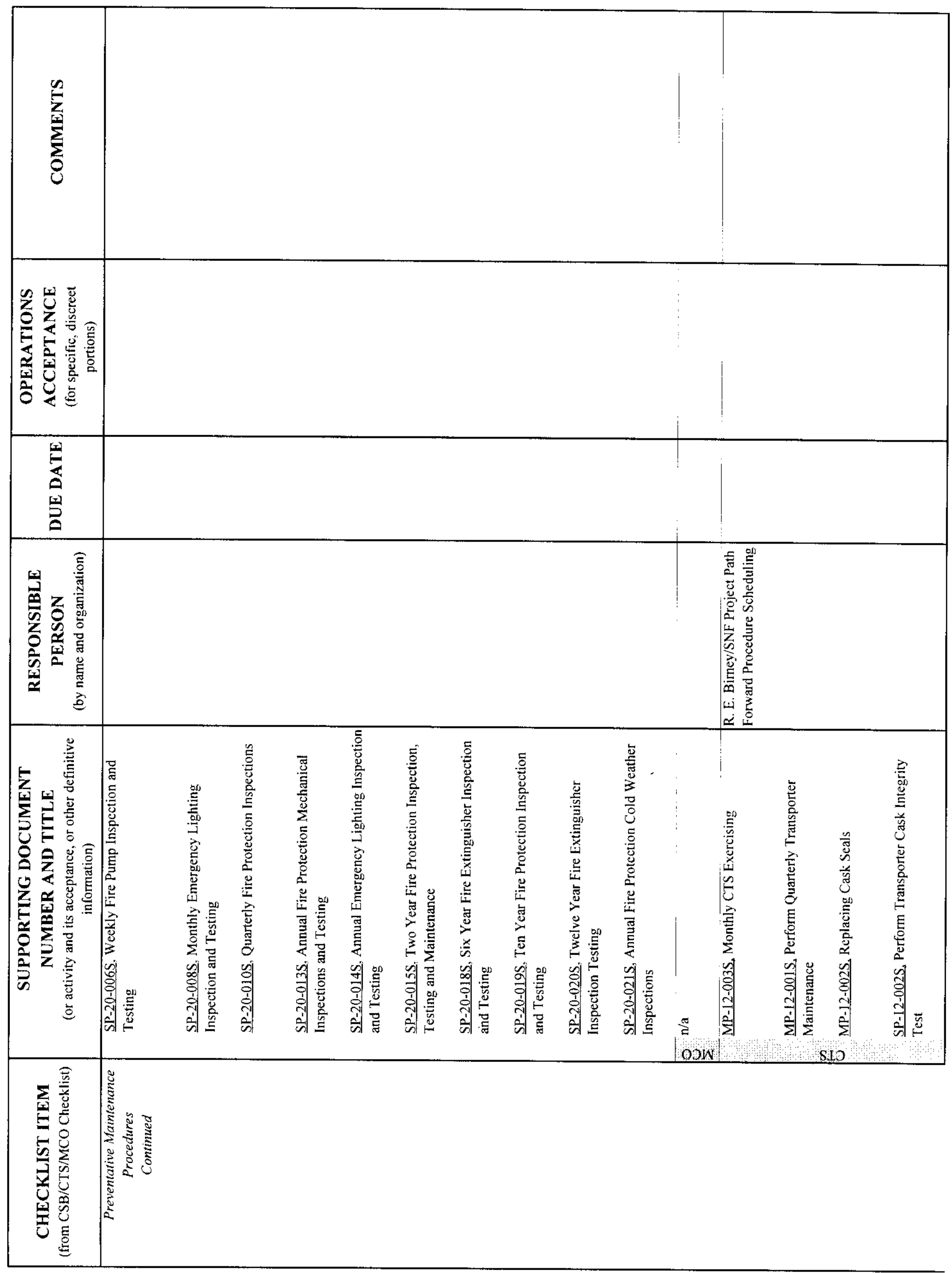


SNF-6253 REV 0

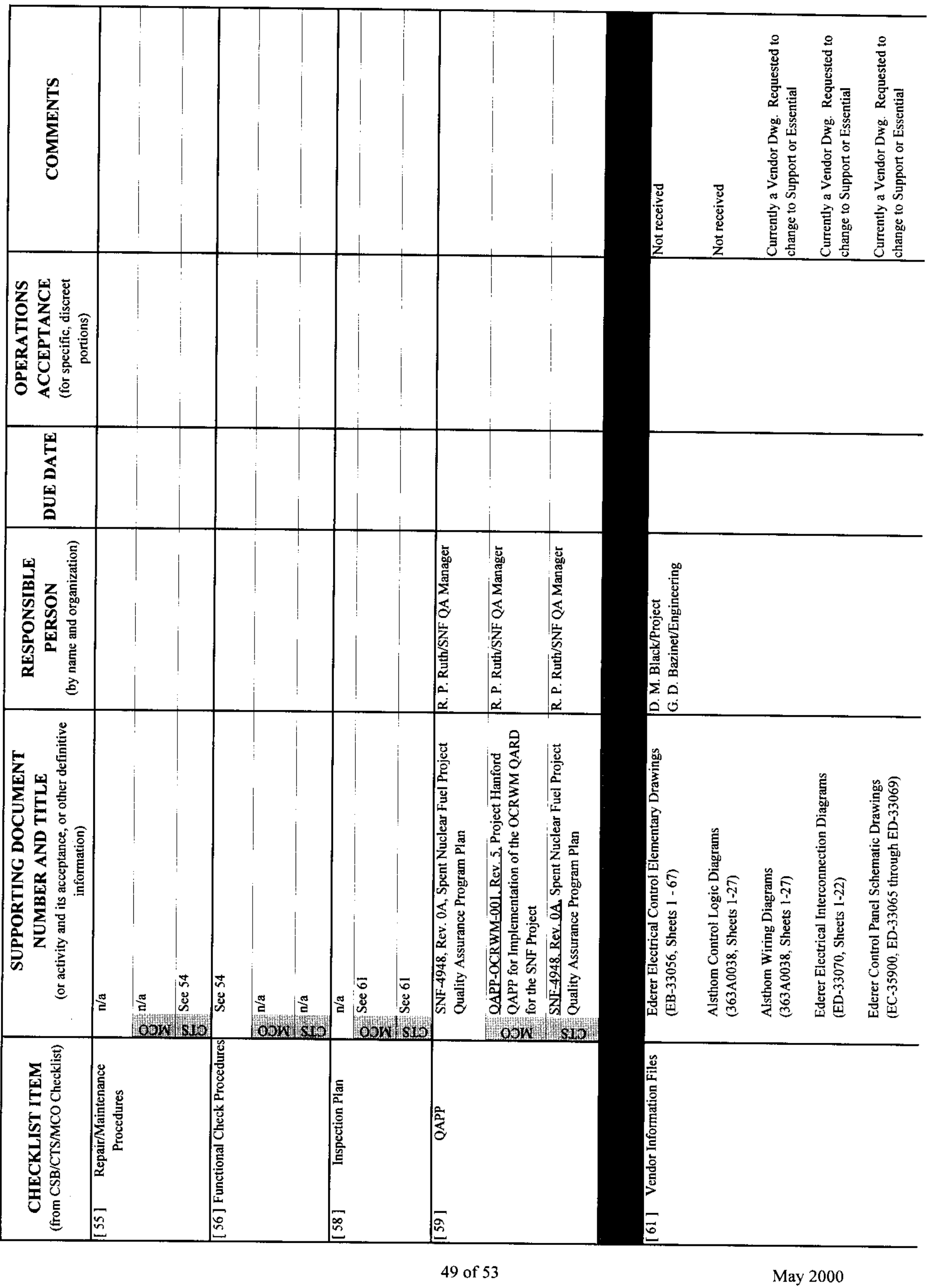


SNF-6253 REV 0

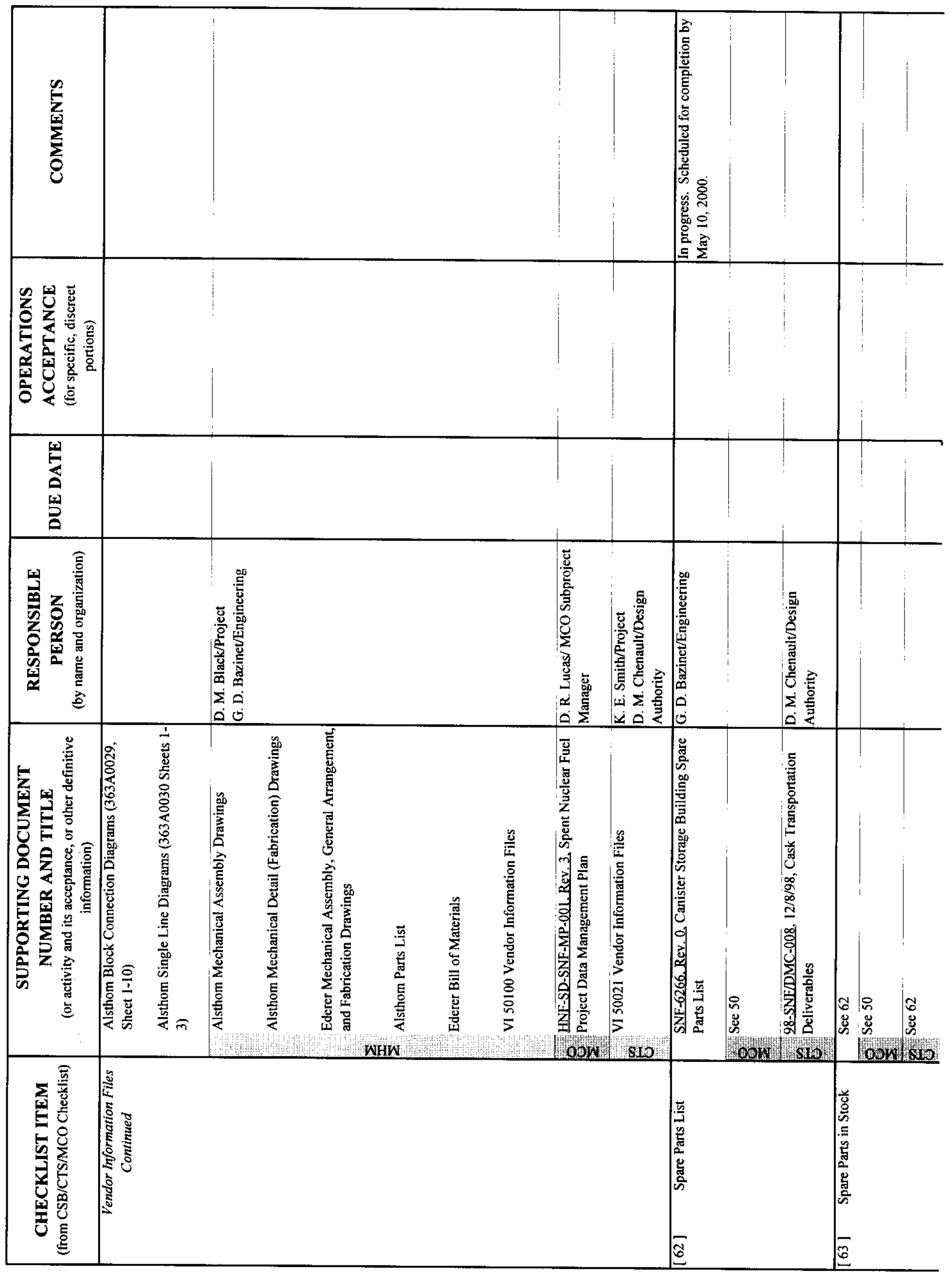


SNF-6253 REV 0

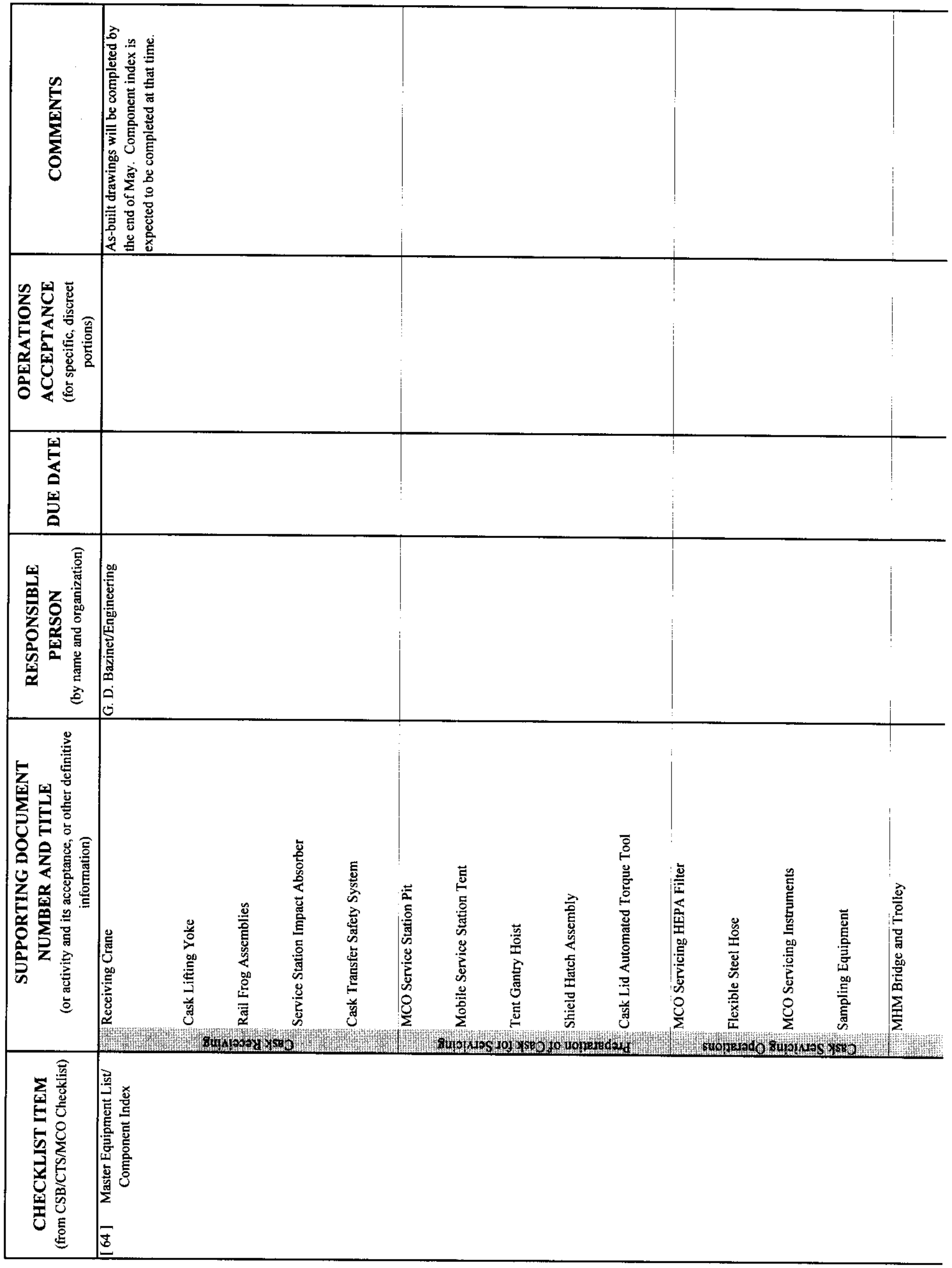


SNF-6253 REV 0

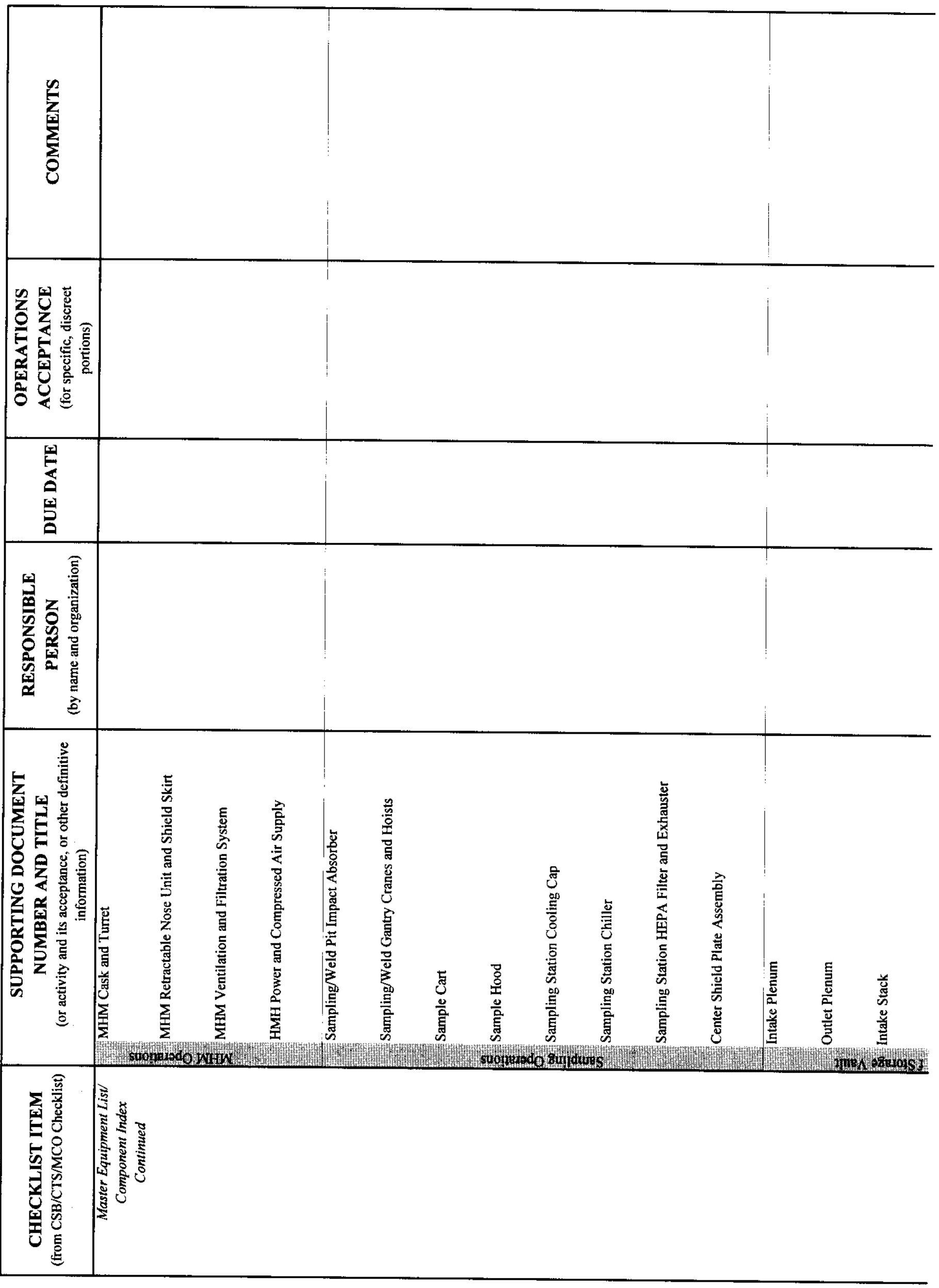


SNF-6253 REV 0

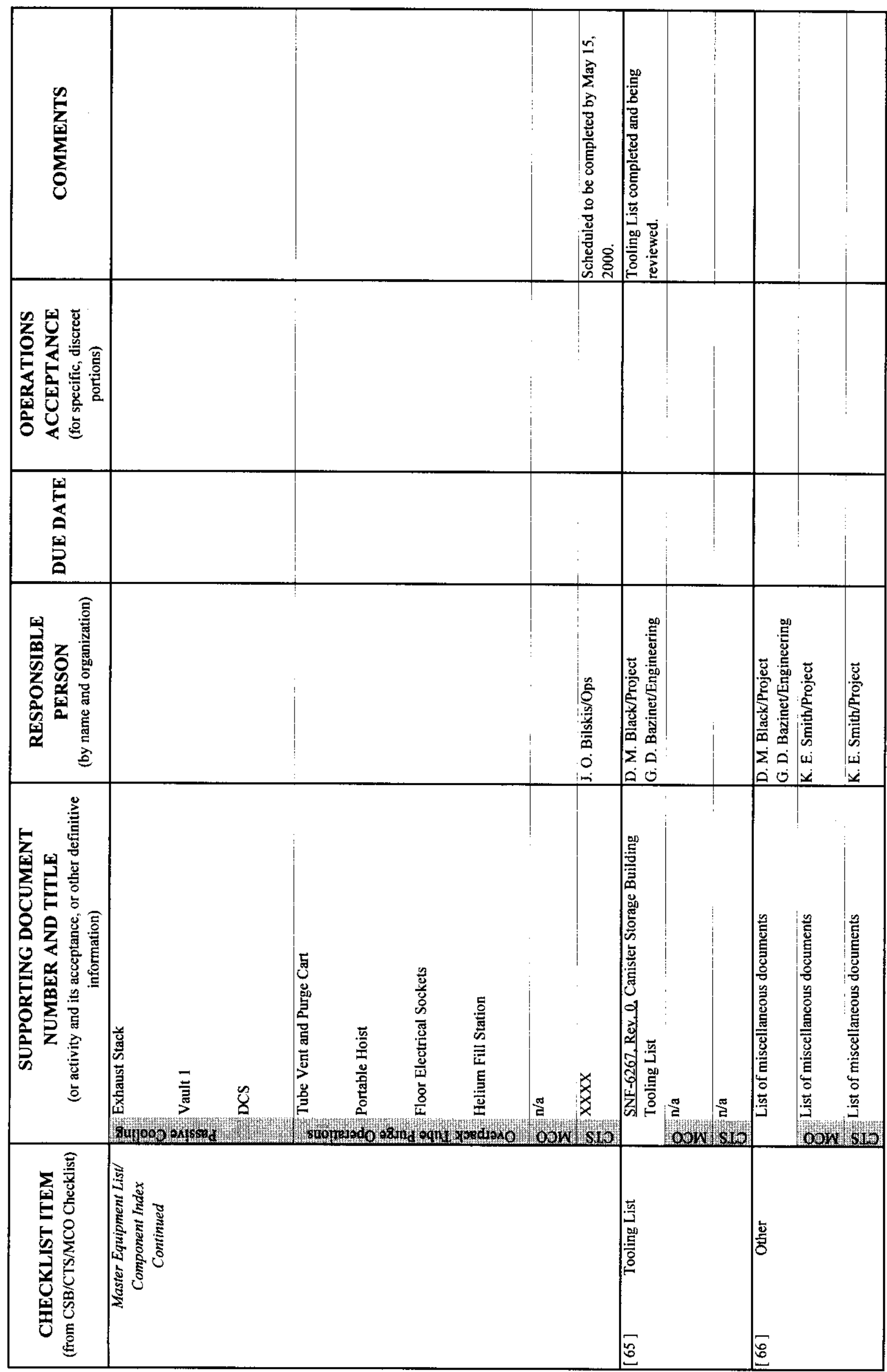




\section{DISTRIBUTION SHEET}

\begin{tabular}{|c|c|c|c|c|c|}
\hline \multirow{2}{*}{ Do } & \multirow{2}{*}{\multicolumn{3}{|c|}{ From }} & \multicolumn{2}{|l|}{ Page 1 of 2} \\
\hline & & & & \multicolumn{2}{|l|}{ Date $5 / 8 / 00$} \\
\hline \multicolumn{4}{|l|}{ Project Title/Work Order } & \multicolumn{2}{|c|}{ EDT No 628696} \\
\hline \multicolumn{4}{|l|}{ SNF-6253, Rev. 0} & \multirow{2}{*}{\multicolumn{2}{|c|}{ ECN No. N/A }} \\
\hline \multicolumn{4}{|c|}{$\begin{array}{l}\text { Canister Storage Building Acceptance for Beneficial Use Index - Roadmap of } \\
\text { Supporting Documents }\end{array}$} & & \\
\hline Name & MSIN & $\begin{array}{l}\text { Text } \\
\text { With All } \\
\text { Attach. }\end{array}$ & Text Only & $\begin{array}{l}\text { Attach./ } \\
\text { Appendix } \\
\text { Only }\end{array}$ & $\begin{array}{c}\text { EDT/ECN } \\
\text { Only }\end{array}$ \\
\hline
\end{tabular}

G. E. Babbitt

G. D. Bazinet

J. O. Bilskis

R. E. Birney

D. M. Black

R. J. Bottenus

D. M. Chenault

A. S. Daughtridge

G. L. Eaton

R. L. Garrett

L. H. Goldmann

P. A. Haine

N. O. Hinojosa

C. R. Hoover

R. J. Jablonski

J. T. Koberg

S. A. Kreig

F. H. Lemieux

B. D. Lorenz

D. R. Lucas

S. S. Moss

T. K. Orgill

R. P. Ruth

O. M. Serrano

K. E. Smith

$\begin{array}{ll}\text { S2-45 } & \text { X } \\ \text { S8-06 } & \text { X } \\ \text { S2-44 } & \text { X } \\ \text { S0-10 } & \text { X } \\ \text { R3-86 } & \text { X } \\ \text { S1-53 } & \text { X } \\ \text { R3-86 } & \text { X } \\ \text { R3-86 } & \text { X } \\ \text { S2-45 } & \text { X } \\ \text { R3-26 } & \text { X } \\ \text { R3-86 } & \text { X } \\ \text { S1-53 } & \text { X } \\ \text { X3-67 } & \text { X } \\ \text { L6-58 } & \text { X } \\ \text { S1-53 } & \text { X } \\ \text { S8-06 } & \text { X } \\ \text { S8-06 } & \text { X } \\ \text { S2-45 } & \text { X } \\ \text { R3-26 } & \text { X } \\ \text { L6-58 } & \text { X } \\ \text { R3-11 } & \text { X } \\ \text { S2-44 } & \text { X } \\ \text { R3-11 } & \text { X } \\ \text { S2-44 } & \text { X } \\ \text { L6-58 } & \text { X }\end{array}$




\section{DISTRIBUTION SHEET}

\section{Continued}

\begin{tabular}{|c|c|c|c|c|c|}
\hline \multirow{2}{*}{$\begin{array}{l}\text { To } \\
\text { Distribution }\end{array}$} & \multirow{2}{*}{\multicolumn{3}{|c|}{$\begin{array}{l}\text { From } \\
\text { CSB Subproject }\end{array}$}} & \multirow{2}{*}{\multicolumn{2}{|c|}{$\frac{\text { Page } 2 \text { of } 2}{\text { Date } 5 / 8 / 00}$}} \\
\hline & & & & & \\
\hline Name & MSIN & $\begin{array}{c}\text { Text } \\
\text { With All } \\
\text { Attach. }\end{array}$ & Text Only & $\begin{array}{l}\text { Attach./ } \\
\text { Appendix } \\
\text { Only }\end{array}$ & $\begin{array}{c}\text { EDT/ECN } \\
\text { Only }\end{array}$ \\
\hline C. E. Swenson & S8-07 & $\mathrm{X}$ & & & \\
\hline B. J. Trawinski & $\mathrm{R} 3-26$ & $\mathrm{X}$ & & & \\
\hline J. E. Turnbaugh & $X 3-79$ & $\mathrm{X}$ & & & \\
\hline R. M. Yanochko & R3-86 & $X$ & & & \\
\hline CSB Project Files & S8-06 & $X$ & & & \\
\hline Start-up Library & $\mathrm{B} 2-64$ & $\mathrm{X}$ & & & \\
\hline
\end{tabular}

